\title{
Polymorphism in Bi-based perovskite oxides: a first-principles study
}

\author{
Akansha Singh, ${ }^{1,2}$ Viveka N. Singh, ${ }^{1}$ Enric Canadell,${ }^{3}$ Jorge Íñiguez, ${ }^{4}$ and Oswaldo Diéguez ${ }^{1,2 *}$ \\ ${ }^{1}$ Department of Materials Science and Engineering, \\ Faculty of Engineering, Tel Aviv University, Tel Aviv 69978, Israel \\ ${ }^{2}$ The Raymond and Beverly Sackler Center for Computational Molecular \\ and Materials Science, Tel Aviv University, Tel Aviv 69978, Israel \\ ${ }^{3}$ Institut de Ciència de Materials de Barcelona (ICMAB-CSIC), Campus UAB, E-08193 Bellaterra, Spain \\ ${ }^{4}$ Materials Research and Technology Department, \\ Luxembourg Institute of Science and Technology, \\ 5 avenue des Hauts-Fourneaux, L-4362 Esch/Alzette, Luxembourg
}

\begin{abstract}
Under normal conditions, bulk crystals of $\mathrm{BiScO}_{3}, \mathrm{BiCrO}_{3}, \mathrm{BiMnO}_{3}, \mathrm{BiFeO}_{3}$, and $\mathrm{BiCoO}_{3}$ present three very different variations of the perovskite structure: an antipolar phase, a rhombohedral phase with a large polarization along the space diagonal of the pseudocubic unit cell, and a supertetragonal phase with even larger polarization. With the aim of understanding the causes for this variety, we have used a genetic algorithm to search for minima in the surface energy of these materials. Our results show that the number of these minima is very large when compared to that of typical ferroelectric perovskites like $\mathrm{BaTiO}_{3}$ and $\mathrm{PbTiO}_{3}$, and that a fine energy balance between them results in the large structural differences seen. As byproducts of our search we have identified charge-ordering structures with low energy in $\mathrm{BiMnO}_{3}$, and several phases with energies that are similar to that of the ground state of $\mathrm{BiCrO}_{3}$. We have also found that a inverse supertetragonal phase exists in bulk, likely to be favored in films epitaxially grown at large values of tensile misfit strain.
\end{abstract}

\section{INTRODUCTION}

Thousands of research articles about $\mathrm{BiFeO}_{3}$ have been published since Wang et al! ${ }^{1}$ reported that epitaxial films of this material were multiferroic at room temperature (Ref. 2 reviews the properties of this oxide). This effort has unvealed potential applications based on the possibility to control the electric polarization with a magnetic field $\mathrm{d}^{3}$ and the magnetization with an electric field ${ }^{4}$ Other phenomena such as domain wall conductivity, ${ }^{[5]}$ novel photovoltaic effects,,$[6$ and the presence of a feature akin to a morphotropic phase boundary in thin films $s^{7}$ have further fuelled research on $\mathrm{BiFeO}_{3}$.

$\mathrm{BiFeO}_{3}$ has a simple crystal structure in bulk at room temperature - a perovskite oxide with 10 atoms in a rhombohedral unit cell and space group $R 3 c{ }^{8}$ The cations are displaced along the space diagonal of the perovskite pseudocubic unit cell, and the $\mathrm{O}_{6}$ octahedra rotate in antiphase about this same three-fold axis, as shown in Fig. 1(a); the pseudocubic lattice constant is $3.965 \AA$ and the pseudocubic angle is $89.45^{\circ} .9$ The large displacements of the cations give rise to a polarization 2 of around $100 \mu \mathrm{C} / \mathrm{cm}^{2}$, while a slight canting of the spins of the $\mathrm{Fe}^{+3}$ ions is responsible for the tiny ferromagnetic moment experimentally measured in an otherwise strong antiferromagnet of the $\mathrm{G}$ type $\mathrm{e}^{2}$ (the type in which the spins of two nearest $\mathrm{Fe}^{+3}$ ions are antiparallel; in bulk $\mathrm{BiFeO}_{3}$ there is in addition a spiral spin wave of period $640 \AA)$.

None of the other bismuth $3 d$ transition-metal per- ovskite insulators displays this $R 3 c$ structure in bulk. $10[11$ $\mathrm{BiCoO}_{3}$ crystallizes in what is called a supertetragonal structure - a tetragonal structure of $P 4 \mathrm{~mm}$ space group where the $c / a$ ratio is so large $(1.27)$ that the $\mathrm{O}_{6}$ octahedra become $\mathrm{O}_{5}$ pyramids, as depicted in Fig. 1(b); every plane of $\mathrm{Co}^{3+}$ ions perpendicular to the $c$ axis has its spin aligned antiparallel to that of its nearest neighbors, while these planes are stacked along $c$ in ferromagnetic fashion $\left(\mathrm{BiCoO}_{3}\right.$ is therefore a C-type antiferromagnet). $\mathrm{BiScO}_{3}$ (a non-magnetic material), $\mathrm{BiCrO}_{3}$ (a G-type antiferromagnet, like $\mathrm{BiFeO}_{3}$ ), and $\mathrm{BiMnO}_{3}$ (the only ferromagnet in this family) have been experimentally reported to crystallize in a centrosymmetric phase with $C 2 / c$ space group, like the one shown in Fig. 1(c). In contrast with this rich behavior under normal conditions, when the temperature or the pressure becomes large enough all these oxides $\left(\mathrm{BiScO}_{3}, \mathrm{BiCrO}_{3}\right.$, $\mathrm{BiMnO}_{3}, \mathrm{BiFeO}_{3}$, and $\mathrm{BiCoO}_{3}$ ) display the same structural phase $\frac{10}{10}$ the paraelectric structure with space group Pnma commonly found in perovksites, $12[13$ represented in Fig. 1(d).

First-principles studies have helped to characterize these structures for each material involved. In particular, they have confirmed that they correspond to special points of the energy surface: the $R 3 c$ structure of $\mathrm{BiFeO}_{3}$ was identified as its ground state according to first-principles calculations already in Ref. 1) McLeod et $a l{ }^{14}$ carried out first-principles optimizations of the $C 2 / c$ structure of $\mathrm{BiScO}_{3}$ and $\mathrm{BiCrO}_{3}$, which showed excellent agreement with experimental results regarding structural 


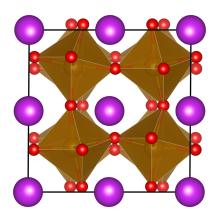

(a) $R 3 \mathrm{c}$

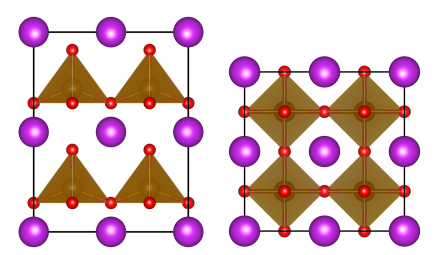

(b) $P 4 m m$
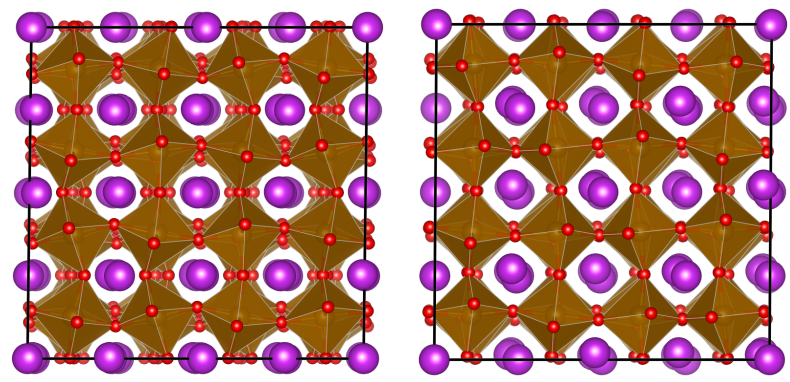

(c) $C 2 / c$

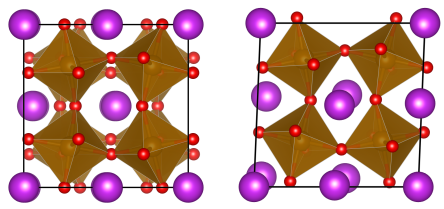

(d) Pnma

FIG. 1. Structures of insulating $\mathrm{BiXO}_{3}$ crystals reported experimentally, labelled according to their corresponding space groups: (a) $\mathrm{BiFeO}_{3}$ at normal conditions, (b) $\mathrm{BiCoO}_{3}$ at normal conditions, (c) $\mathrm{BiScO}_{3}, \mathrm{BiCrO}_{3}$, and $\mathrm{BiMnO}_{3}$ at normal conditions, and (d) $\mathrm{BiFeO}_{3}, \mathrm{BiScO}_{3}, \mathrm{BiCrO}_{3}, \mathrm{BiMnO}_{3}$, and $\mathrm{BiCoO}_{3}$ at high temperature or high pressure. We plot the smallest pseudocubic supercell compatible with each phase; here and in later figures of this kind we show views along the $x, y$, and $z$ pseudocubic axes that are inequivalent (when only two pictures are included, the left picture corresponds to the two views that are equivalent).

parameters; Baettig, Seshadri, and Spaldir 150 confirmed the $C 2 / c$ structure as the ground state of $\mathrm{BiMnO}_{3}$; and the supertetragonal structure of bulk $\mathrm{BiCoO}_{3}$ was first analyzed from the first-principles point of view in Ref. 16, where a large value of its polarization was predicted. Computational studies also probed phases other than the ground state; early examples of these concern $\mathrm{BiCrO}_{3} 17$ (showing that it is possible to optimize a phase with the structure of bulk $\mathrm{BiFeO}_{3}$ ) and $\mathrm{BiFeO}_{3}{ }^{18}$ (where the $R 3 c$ and four other additional phases were optimized). In our previous work, we reported that the energy surface of bulk $\mathrm{BiFeO}_{3}$ shows many local minima, including several of supertetragonal type. $\frac{19}{19}$ We also showed that in $\mathrm{BiMnO}_{3}$ there are local minima of the bulk energy in addition to the experimental $C 2 / c$ ground state,${ }^{20}$ and that in $\mathrm{BiCoO}_{3}$ the orthorhombic Pnma phase and a $R 3 c$-like phase have low energy ${ }^{21}$ Finally, let us note that there is a considerable amount of work on solid solutions, particularly lanthanide-doped $\mathrm{BiFeO}_{3}{ }^{22}$ Beyond providing alternatives to tune the properties of bismuth ferrite, e.g. by inducing morphotropic phases boundaries 2325 these solid solutions often feature long-period structures involving unusual rotation patterns of the $\mathrm{O}_{6}$ octahedra 26

It is thus abundantly clear that Bi-based perovskite oxides display a polymorphism that renders them a unique and very attractive materials family. In view of this, it is natural to wonder whether these materials have already revealed to us all the structures they have in store, or whether they may present even more phases with unexpected features. At the same time, it is not yet clear whether the polymorphism affects all Bi-based perovskite oxides, whether the presence of specific transition metal cations may play a critical role, whether a similar structural richness is present (hiding) in other perovskites, etc. In this article we address these issues by running a systematic search for bulk metastable phases of $\mathrm{Bi} X \mathrm{O}_{3}$ compounds $(X=\mathrm{Sc}, \mathrm{Cr}, \mathrm{Mn}, \mathrm{Fe}, \mathrm{Co})$. We do this in an automatic and unbiased way with the help of the USPEX $\left.{ }^{27} 29\right]$ evolutionary algorithm for the search of crystal structures. We show that these oxides show very similar features in their energy surfaces, with the same structures appearing as minima in many of the materials, and that small energy differences between those minima are responsible for the rich variety of polymorphs displayed. We also compare this situation with what we obtain for some of the most used ferroelectric perovskite oxides $\left(\mathrm{BaTiO}_{3}\right.$ and $\left.\mathrm{PbTiO}_{3}\right)$ where very few minima exist in their energy surface. In Section II we describe in detail the Methodology that we have used. In Section III we present and discuss our results. Finally, we state our conclusions in Section IV.

\section{METHOD}

We used the vasP software ${ }^{30}$ to carry out calculations based on density-functional theory (DFT)! ${ }^{3132}$ Since DFT does not reproduce well the localization of $3 d$ electrons in some of these oxides, a correction inspired in the Hubbard model was added; this methodology is usually refered to as $\mathrm{LDA}+U$ or $\mathrm{DFT}+U, \underline{33}$ (details about it are given in Table I). We approximated the exchange-correlation functional following the work of Perdew, Burke, and Ernzerhof adapted to solids (PBEsol); different exchange-correlation approximations can affect the energy difference between minima of the energy surface, but in most cases they agree regarding whether a crystal structure is a minimum of the energy or not. ${ }^{[19}$ To capture the interaction between the valence electrons and the ion cores we used projector-augmented wave (PAW) potentials. ${ }^{37 / 38}$ The electrons treated as valence ones were: $3 p, 3 d$, and $4 s(\mathrm{Sc}, \mathrm{Ti}, \mathrm{Cr}, \mathrm{Mn}, \mathrm{Fe}$, and $\mathrm{Co}) ; 5 s, 5 p, 6 s(\mathrm{Ba}) ; 5 d, 6 s$, and $6 p(\mathrm{~Pb}) ; 5 d$ and $6 s(\mathrm{Bi})$; and $2 s$ and $2 p(\mathrm{O})$.

In order to carry out an unbiased search for energy 
TABLE I. Details of the DFT $+U$ methodology used to treat the $d$ electrons of the transition-metal atoms: formalism references, values of the effective on-site Coulomb interactions $U$, and values of the effective on-site exchange interactions $J$. Those values are chosen based on previous experience; our tests showed that varying these parameters within a few eV has a small effect on whether a configuration is a minimum of the energy or not, and on the variation of the value of bond lengths and lattice parameters $\stackrel{36}{{ }^{36}}$ Regular DFT (no $U$ ) was used to treat $\mathrm{BiScO}_{3}, \mathrm{BaTiO}_{3}$, and $\mathrm{PbTiO}_{3}$.

\begin{tabular}{rcccc}
\hline \hline & $\mathrm{BiCrO}_{3}$ & $\mathrm{BiMnO}_{3}$ & $\mathrm{BiFeO}_{3}$ & $\mathrm{BiCoO}_{3}$ \\
\hline Formalism Ref. 34 & Ref. 35 & Ref. 34 & Ref. 34 \\
$U$ & $2.2 \mathrm{eV}$ & $4.0 \mathrm{eV}$ & $4.0 \mathrm{eV}$ & $6.0 \mathrm{eV}$ \\
$J$ & $0.0 \mathrm{eV}$ & $1.0 \mathrm{eV}$ & $0.0 \mathrm{eV}$ & $0.0 \mathrm{eV}$ \\
\hline \hline
\end{tabular}

minima we have used the USPEx $27 \sqrt[29]{29}$ code, which implements an evolutionary algorithm to find crystal structures with low energy. We worked with structures that have 10 or 20 atoms in their unit cell; the initial space group and atomic positions were chosen randomly by the code. For every USPEX run we generated 50 of such random structures, and each of them was optimized by VASP using first a conjugate-gradient algorithm and then a quasi-Newton algorithm. 39 The energy of the resulting configurations was taken as a fitness parameter to qualify for the next generation of trial structures, composed of 30 of them (this was also the number of structures used in subsequent generations). From then on, the population in every generation was computed following USPEX' specially designed variational operators (heredity, mutation, and soft-mutation) ${ }^{29}$ This procedure was stopped at a maximum of 35 generations, or when the energy of the best structure did not change for 15 consecutive generations. The calculations were performed at different degrees of numerical convergence, with the most accurate level of calculations using a plane-wave energy cutoff of $500 \mathrm{eV}$, a reciprocal space resolution of $0.06 \times 2 \pi \AA^{-1}$, and a force stopping criterion of $0.01 \mathrm{eV} / \AA$. All the structures obtained in this way were reoptimized using an energy cutoff of $600 \mathrm{eV}$, a self-consistent cycle energy threshold of $10^{-8} \mathrm{eV}$, and a force stopping criterion of 0.001 $\mathrm{eV} / \AA$. The zone centered ( $\Gamma$-point) frequencies were also calculated using finite differences, to ensure that the obtained structures are local minima of the energy in the simulation box used.

Once a structure was identified by the USPEX search as a local minimum of the energy for one of the materials, we optimized it also for the other materials (starting with a configuration where the lattice parameters were rescaled to take into account that the ion sizes are differents for different oxides). While in some cases UsPEx found the same structures in different materials, in a few cases these new optimizations identified new energy minima.

As mentioned earlier, the Bi-based oxides studied here show different magnetic orderings: ferromagnetism $\left(\mathrm{BiMnO}_{3}\right)$, G-type antiferromagnetism $\left(\mathrm{BiCrO}_{3}\right.$ and
$\left.\mathrm{BiFeO}_{3}\right)$, C-type antiferromagnetism $\left(\mathrm{BiCoO}_{3}\right)$, and zero magnetic moments $\left(\mathrm{BiScO}_{3}\right)$. In our study with USPEX we used antiferromagnetic 10- and 20-atom unit cells where the initial spins were assigned randomly. After we found crystal structures that were a minimum of the energy, we enlarged (if needed) and reoptimized the cell, so that the energy of other typical magnetic orderings was also computed. In our previous studies in some these perovskites we found that once one type of magnetic ordering is a minimum of the energy, others also exist as minima of the energy $\frac{19}{21}$

Finally, we resorted to hybrid calculations for the few cases where we required a more accurate calculation of the energy differences within a group of phases, or a more accurate estimation of bandgaps. We used the

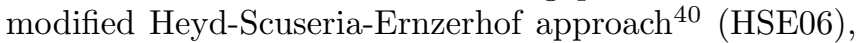
which predicts bandgaps in good agreement with experiment for perovskite oxides $\frac{41}{4}$; contrary to $\mathrm{DFT}+U$, it also predicts the ground state of $\mathrm{BiMnO}_{3}$ to be the experimentally identified one ${ }^{20}$ Calculations with this method are two orders of magnitude slower than with $\mathrm{DFT}+U$, but, apart from the better agreement with experiment, they do not require differente fitting parameters for each material (HSE06 uses a fraction of exact exchange equal to $25 \%$, and a range-separation parameter equal to $0.20 \AA^{-1}$ ).

Note that, because we choose to work with relatively small cells, this investigation does not address the longperiod polymorphs that, as mentioned above, are believed to occur in some $\mathrm{BiFeO}_{3}$-based solid solutions. Yet, as we will see, even if restricted to relatively small cell sizes, our simulations reveal an incredible structural richness that clearly single out Bi-based perovskites from the rest.

\section{RESULTS}

First, we report in detail the $\mathrm{BiFeO}_{3}$ polymorphs identified during our evolutionary-algorithm search. Despite of the extensive previous studies carried out for this oxide, we have identified several structures with low energy that had not been reported so far as energy minima of $\mathrm{BiFeO}_{3}$. We then move on to the results of a similar search for other Bi-based transition-metal oxides; these harbor similar polymorphs to those of $\mathrm{BiFeO}_{3}$, but slight differences in energy between those polymorphs lead to global minima that are structurally very different. We also report similar searches for prototype perovskites $\mathrm{BaTiO}_{3}$ and $\mathrm{PbTiO}_{3}$ for comparison. In the following three subsections we give more details about novel results of our search: structures with energies very close to that of the ground state of $\mathrm{BiScO}_{3}, \mathrm{BiCrO}_{3}$, and $\mathrm{BiMnO}_{3}$; charge-ordering phases of $\mathrm{BiMnO}_{3}$; and details about a polar phase expected to be stable as an epitaxial film is some cases. Finally, we report bandgap trends for the different phases found, and we discuss the reasons for the variety of polymorphs predicted for Bi-based perovskite 
oxides.

\section{A. Local minima of $\mathrm{BiFeO}_{3}$}

Our searches for polymorphs of $\mathrm{BiFeO}_{3}$ with USPEX following the procudure describe in the Methods Section produced hundreds of structures, a result that is consistent with our initial exploration based on a more rudimentary approach $[19]$ In order to sort out which of those structures were unique, we used the crystal fingerprint method described in Ref. 42, this allowed us to identify duplicated structures that differ slightly in their atomic coordinate positions due to numerical noise, and structures that differ only because of the use of different unit cell shapes and sizes by USPEX. The structures that remained were re-optimized in the G- and C-type antiferromagnetic patterns. Only the lowest-energy magnetic ordering is reported here.

In this way we found 17 structurally unique structures within less than $200 \mathrm{meV} /$ f.u. of the ground state. These local minima within 10- and 20-atom unit cells are listed in Table I. The ground state is, in agreement with experiment, the polar $R 3 c$ phase with G-type antiferromagnetism. The next configuration in energy is the nonpolar Pnma phase; as mentioned earlier, this is a phase that appears at high temperature or pressure for Bi-based transition-metal perovskite oxides.

A first surprising result appears next in Table II as we identified a polymorph of $\mathrm{BiFeO}_{3}$ with relatively low energy that corresponds to the experimentally reported crystal structure of bulk $\mathrm{BiScO}_{3}, \mathrm{BiCrO}_{3}$, and $\mathrm{BiMnO}_{3}$ in normal conditions. This is a structure with $C 2 / c$ space group in which the pattern of antiparallel $\mathrm{Bi}$ displacements in the planes perpendicular to the inequivalent axis resemble that of $\mathrm{Pb}$ atoms in $\mathrm{PbZrO}_{3}$ (space group $P b a m)$, the prototype of antiferroelectric material. ${ }^{43} \mathrm{In}$ both structures there is an underlying pattern of $\mathrm{O}_{6}$ rotations in antiphase along the [110] direction $\left(a^{-} a^{-} c^{0}\right.$ rotations in Glazer's notation ${ }^{44}$ ). However, as Figure 2 shows, the pattern of $A$ cation displacements in the direction perpendicular to the page is different in the $C 2 / c$ structure and in the $\mathrm{PbZrO}_{3}$ structure - the former is associated with point $(2 \pi / a)(1 / 4,1 / 4,1 / 4)$ of the Brillouin zone of the simple cubic perovskite cell, while the latter it is associated with the $(2 \pi / a)(1 / 4,1 / 4,0)$ point; for $C 2 / c$ $\mathrm{BiFeO}_{3}$ the movement of the $\mathrm{Bi}$ atoms distorts further the $\mathrm{O}_{6}$ octahedra.

The similarity with the antiferroelectric phase of $\mathrm{PbZrO}_{3}$ suggest an intriguing possibility: that, if stabilized, the non-polar polymorphs of $\mathrm{BiFeO}_{3}$ would probably behave as antiferroelectrics $\stackrel{45}{4}$ as it seems likely that they could be transformed, by application of an electric field, into the polar $R 3 c$ state. This is in fact a behavior that is being explored in $\mathrm{BiFeO}_{3}$ solid solutions in which the Pnma structure exists at ambient conditions, $\frac{46 \mid 47}{\text { our }}$ results suggest that other antiferroelectric phases could be similarly identified. (A precise definition of an antifer- roelectric phase is a matter of some debate; it is not our purpose here to contribute to that debate, and we simply adopt the practical view point just introduced. Also, in the following, when referring to atomic displacement patterns like those of $\mathrm{Pb}$ in the $\mathrm{PbZrO}_{3}$ structure, we use the terms antiferroelectric and antipolar indistinctly.)

When we optimized $\mathrm{BiFeO}_{3}$ starting with a structure like that of $\mathrm{PbZrO}_{3}$ we did not obtain a minimum of the energy - instead, the relaxed structure shows a soft mode that involves additional antiferroelectric displacements of the $\mathrm{Bi}$ atoms along the inequivalent axis; if we distort the structure along that mode, the energy indeed lowers, and re-optimization leads to a 40-atom unit cell phase around $41 \mathrm{meV} / \mathrm{fu}$ above the ground state with $P 2_{1} / c$ symmetry (which could not have been found in our USPEX search since it has a 40-atom primitive cell).

Complex patterns of antiferroelectric Bi displacements appear also in the next most stable structure found, of space group $P \overline{1}$ (Figure 3(a)). Like the $C 2 / c$ phase, this low-symmetry non-polar structure shows an antiferroelectric pattern tied to the $(2 \pi / a)(1 / 4,1 / 4,1 / 4)$ point of the first Brillouin zone, and a $a^{-} b^{-} c^{-}$rotation pattern for the $\mathrm{O}_{6}$ octahedra. Another difference with the $C 2 / c$ phase is that now the Bi atoms move substantially along the three pseudocubic axes.

The four lowest energy structures of Table II have in common that their three pseudocubic lattice parameters are similar in value. As discussed in our previous work, $\stackrel{49}{4}$ this implies that the favoured antiferromagnetic ordering is of the G type. Polar structures 12, 13, 15, and 16, and antipolar structure 17 also fit this pattern, although Fig. 3 shows how diverse they are in their geometries.

For example, in structure 12 (space group $P c$, Fig. 3(b)) we find a mix of a polar $\Gamma_{4}^{-}$mode along (111) with and antipolar $M_{5}^{-}$mode along (110), resulting in large equal $\mathrm{Bi}$ displacements along the $z$ pseudocubic axis, and displacements of $\mathrm{Bi}$ atoms in the perpendicular plane that involve half the atoms moving along the $x$ pseudocubic axis and the other half moving along the $y$ pseudocubic axis; the associated polarization lies close to the (111) pseudocubic axis, while the $\mathrm{O}_{6}$ octahedra show a $a^{0} a^{0} c^{+}$pattern.

Structure 13 (space group $\mathrm{Cm}$, Fig. 3(c)) also mixes a polar and an antipolar mode: a $\Gamma_{4}^{-}$mode along with components along the $x$ and $z$ directions, and a $M_{5}^{-}$mode almost perpendicular to it; this results in a complex pattern of Bi displacements along two different pseudocubic axes and the corresponding in-plane polarization, together with a $\left(a^{+} b^{+} c^{+}\right) \mathrm{O}_{6}$ rotation.

Structure 15 (space group $P m c 2_{1}$, Fig. 3(d)) is far from a typical perovskite; the coexistence of a $\Gamma_{4}^{-}$component along (110) with a strong $M_{5}^{-}$mode perpendicular to it results in the breaking of the $\mathrm{O}_{6}$ octahedra into $\mathrm{O}_{5}$ pyramids, but these pyramids form a zig-zag pattern different to the one in supertetragonal structures. This structure has been reported before ${ }^{50}$ as a plausible phase to appear in epitaxial films under large tensile strains; now we see that it is a minimum in bulk $\mathrm{BiFeO}_{3}$, giving sup- 
TABLE II. $\mathrm{BiFeO}_{3}$ phases found by USPEx (local minima of the energy in 10-atom or 20-atom cells). Column 1: phase index. Column 2: space group. Column 3: favoured type of antiferromagnetism. Column 4: energy difference with the ground state (in meV per formula unit). Columns 5-10: lattice constants (in $\AA$ ) and lattice angles (in degrees). Column 11: polarization arising from polar Bi displacements within the $\Gamma_{4}^{-}$soft-mode of the simple cubic cell (larger polarization components are denoted as $P$; smaller as $p$ ). Column 12: antipolar modes (point of the first Brillouin zone and direction of displacement of half the Bi cations; the other half move in the opposite direction). Column 13: $\mathrm{O}_{6}$ octahedra rotation according to Glazer's notation. ${ }^{44}$ We used the Isotropy software suite $\frac{48}{40}$ obtain the distortions listed here.

\begin{tabular}{|c|c|c|c|c|c|c|c|c|c|c|c|c|}
\hline \multirow[t]{2}{*}{$\#$} & \multirow[t]{2}{*}{ Group } & \multirow{2}{*}{\multicolumn{2}{|c|}{$\mathrm{AFM} \Delta E$}} & \multicolumn{6}{|c|}{ Pseudocubic lattice parameters } & \multicolumn{3}{|c|}{ Significant distortions } \\
\hline & & & & $a$ & $b$ & $c$ & $\alpha$ & $\beta$ & $\gamma$ & Bi (polar) & Bi (antipolar) & $\mathrm{O}$ (rotation) \\
\hline 1 & $R 3 c$ & $\mathrm{G}$ & 0 & 3.945 & 3.945 & 3.945 & 89.58 & 89.58 & 89.58 & $\left(P_{x}, P_{x}, P_{x}\right)$ & - & $\left(a^{-} a^{-} a^{-}\right)$ \\
\hline 2 & Pnma & $\mathrm{G}$ & 28 & 3.914 & 3.914 & 3.885 & 90 & 90 & 87.64 & - & $(2 \pi / a)(0,0,1 / 2),[a a 0]$ & $\left(a^{-} a^{-} c^{+}\right)$ \\
\hline 3 & $C 2 / c$ & $\mathrm{G}$ & 68 & 3.950 & 3.950 & 3.902 & 89.31 & 89.31 & 89.60 & - & $(2 \pi / a)(1 / 4,1 / 4,1 / 4),[a a 0]$ & $\left(a^{-} a^{-} c^{0}\right)$ \\
\hline 4 & $P \overline{1}$ & G & 93 & 3.924 & 3.897 & 3.957 & 89.56 & 89.33 & 89.94 & - & $(2 \pi / a)(1 / 4,1 / 4,1 / 4),[a b c]$ & $\left(a^{-} b^{-} c^{-}\right)$ \\
\hline 5 & $C c$ & $\mathrm{C}$ & 97 & 3.760 & 3.760 & 4.721 & 88.02 & 88.02 & 89.99 & $\left(p_{x}, p_{x}, P_{z}\right)$ & $(2 \pi / a)(1 / 2,1 / 2,1 / 2),[a \bar{a} 0]$ & $\left(a^{-} a^{-} c^{0}\right)$ \\
\hline 6 & $\operatorname{Pna} 2_{1}$ & $\mathrm{C}$ & 100 & 3.756 & 3.756 & 4.722 & 90 & 90 & 89.98 & $\left(0,0, P_{z}\right)$ & $(2 \pi / a)(0,0,1 / 2),[a a 0]$ & $\left(a^{-} a^{-} c^{0}\right)$ \\
\hline 7 & $\mathrm{Cm}$ & $\mathrm{C}$ & 103 & 3.689 & 3.803 & 4.769 & 86.59 & 90 & 90 & $\left(0, p_{y}, P_{z}\right)$ & $(2 \pi / a)(1 / 2,0,1 / 2),[0 a 0]$ & $\left(a^{0} b^{+} c^{0}\right)$ \\
\hline 8 & $C c$ & $\mathrm{C}$ & 105 & 3.747 & 3.753 & 4.741 & 90 & 87.97 & 90 & $\left(p_{x}, 0, P_{z}\right)$ & $(2 \pi / a)(0,0,1 / 2),[0 a 0]$ & $\left(a^{0} b^{-} c^{+}\right)$ \\
\hline 9 & $P c$ & $\mathrm{C}$ & 106 & 3.750 & 3.750 & 4.744 & 88.09 & 88.09 & 89.74 & $\left(p_{x}, p_{x}, P_{z}\right)$ & $(2 \pi / a)(1 / 2,1 / 2,0),[a \bar{a} 0]$ & $\left(a^{0} a^{0} c^{+}\right)$ \\
\hline 10 & $\operatorname{Pmn} 2_{1}$ & $\mathrm{C}$ & 106 & 3.690 & 3.799 & 4.762 & 90 & 90 & 90 & $\left(0,0, P_{z}\right)$ & $(2 \pi / a)(0,0,1 / 2),[0 a 0]$ & $\left(a^{0} b^{+} c^{0}\right)$ \\
\hline 11 & $\mathrm{Cm}$ & $\mathrm{C}$ & 109 & 3.742 & 3.742 & 4.760 & 87.97 & 87.97 & 89.68 & $\left(p_{x}, p_{y}, P_{z}\right)$ & - & - \\
\hline 12 & $P c$ & G & 109 & 3.987 & 3.987 & 3.971 & 89.06 & 89.06 & 89.92 & $\left(P_{x}, P_{x}, P_{z}\right)$ & $(2 \pi / a)(1 / 2,1 / 2,0),[a \bar{a} 0]$ & $\left(a^{0} a^{0} c^{+}\right)$ \\
\hline 13 & $\mathrm{Cm}$ & G & 111 & 3.915 & 3.928 & 3.982 & 90 & 89.38 & 90 & $\left(P_{x}, 0, P_{z}\right)$ & $(2 \pi / a)(0,1 / 2,1 / 2),[a 0 c]$ & $\left(a^{+} b^{+} c^{+}\right)$ \\
\hline 14 & $F m m 2$ & $\mathrm{C}$ & 116 & 3.747 & 3.753 & 4.675 & 90 & 90 & 90 & $\left(0,0, P_{z}\right)$ & $(2 \pi / a)(1 / 2,1 / 2,1 / 2),[a 00]$ & $\left(a^{-} b^{0} c^{0}\right)$ \\
\hline 15 & $P m c 2_{1}$ & G & 119 & 4.189 & 4.189 & 3.706 & 90 & 90 & 88.65 & $\left(P_{x}, P_{x}, 0\right)$ & $(2 \pi / a)(1 / 2,1 / 2,0),[a \bar{a} 0]$ & $\left(a^{0} a^{0} c^{+}\right)$ \\
\hline 16 & $C m$ & G & 125 & 3.987 & 4.031 & 3.871 & 90 & 90 & 89.18 & $\left(P_{x}, P_{y}, 0\right)$ & $(2 \pi / a)(0,1 / 2,1 / 2),[a 00]$ & $\left(a^{+} b^{0} c^{0}\right)$ \\
\hline 17 & $R \overline{3}$ & G & 164 & 3.934 & 3.934 & 3.934 & 89.57 & 89.57 & 89.57 & - & $(2 \pi / a)(1 / 2,1 / 2,1 / 2),[a a a]$ & $\left(a^{-} a^{-} a^{-}\right)$ \\
\hline
\end{tabular}

port to its possible experimental realization even at the nominally large strains required if comparing to the $R 3 c$ ground state. This $P m c 2_{1}$ phase shows the smallest $c / a$ ratio among the $\mathrm{BiFeO}_{3}$ polymorphs, so in the following we refer to it as an inverse supertetragonal structure.

Structure 16 (space group $C m$, Fig. 3(e)) mixes a polar $\Gamma_{4}^{-}$mode in the $x y$ plane with an antipolar $M_{3}^{-}$mode along $x$, resulting in a net polarization off the (110) direction.

Finally, structure 17 (space group R3, Fig. 3(e)) is the antipolar equivalent of the $R 3 c$ ground state in that for every Bi that moves along (111), its six nearest Bi neighbors move along $(\overline{1} \overline{1} \overline{1})$, while the $\mathrm{O}_{6}$ octahedra pattern is still $\left(a^{-} a^{-} a^{-}\right)$.

The rest of phases found by USPEX are of supertetragonal type. Structures 5, 6, 7, and 8 where already found in our previous search, $\frac{19}{19}$ while structures $9,10,11$, and 14 are of a similar kind. All these supertetragonal structures show a large $\Gamma_{4}^{-}$soft mode distortion along $z$, and some of them show polar Bi displacements in the $x y$ plane $(5,7,8,9$, and 11$)$. They differ also regarding slight $\mathrm{O}_{5}$ pyramid rotations.

\section{B. $\mathrm{BiXO} \mathrm{O}_{3}$ Phase Search}

To help understand the complexity of $\mathrm{BiFeO}_{3}$ 's potential energy landscape we compared it to that of related oxides $\mathrm{BiXO}_{3}$, with $X=\mathrm{Sc}, \mathrm{Cr}, \mathrm{Mn}, \mathrm{Co}$. We run UsPEX on 10-atom and 20-atom unit cells of $\mathrm{BiScO}_{3}$ (spin unpolarized calculations), $\mathrm{BiCrO}_{3}$ and $\mathrm{BiCoO}_{3}$ (antiferromagnetic), and $\mathrm{BiMnO}_{3}$ (both ferromagnetic and antiferromagnetic). In order to find which magnetic order is favored, we re-optimized all minima identified by imposing ferromagnetic and and A-type antiferromagnetic for $\mathrm{BiMnO}_{3}$, and $\mathrm{G}$ - and C-type for $\mathrm{BiCrO}_{3}$ and $\mathrm{BiCoO}_{3}$ (these are expected to be the competitive cases $19 \mid 20$ ).

This search resulted in 22 new minima, to add to the 17 initial ones of $\mathrm{BiFeO}_{3}$. The main information about these 39 local minima (in their 10- or 20-atom unit cell) is contained in Table III] where their energy is underlined; this energy is computed with respect to the latest experimental ground states reported. Once a structure was found for one of the transition metals, we re-optimized it for the others too; if this led us to a energy minimum of similar geometric characteristics, we quote its energy, space group, and magnetic ordering in Table III (not underlined). For all calculations reported in this Table we extended the unit cell of the structures to a 40 -atom unit cell-the $2 \times 2 \times 2$ pseudocubic one when 


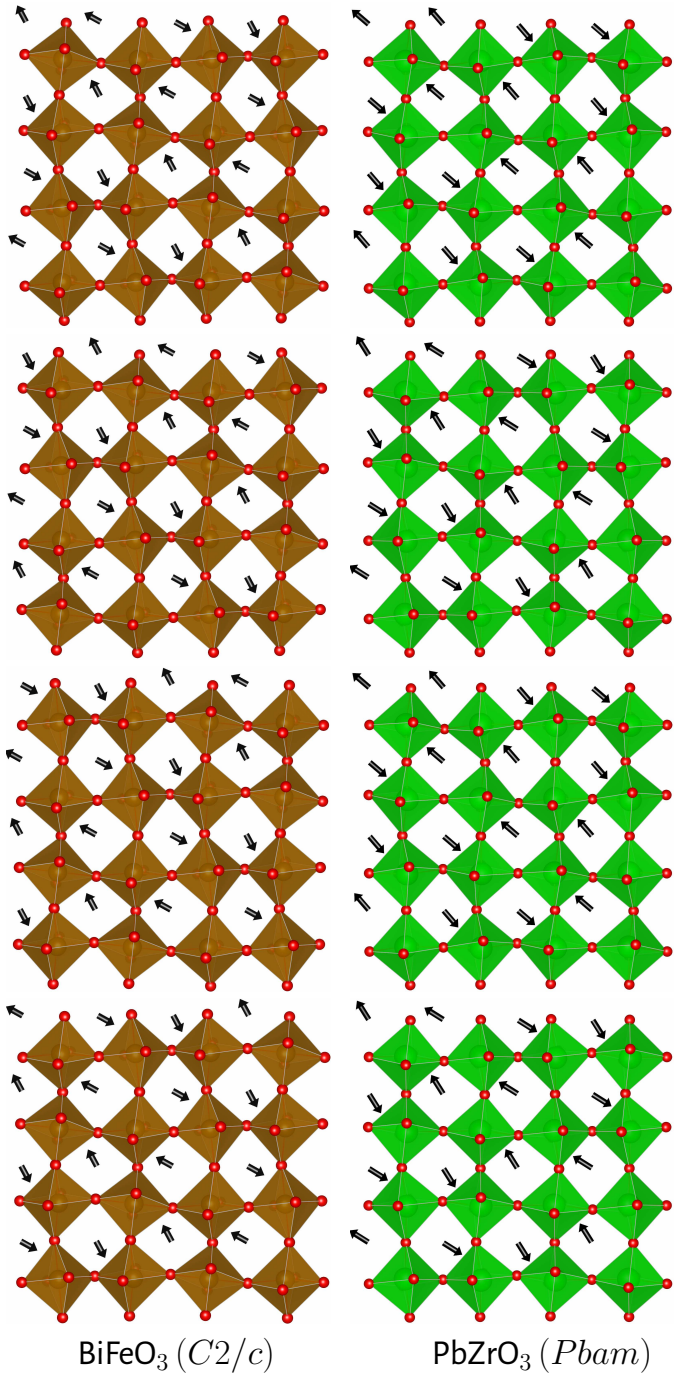

FIG. 2. Comparison between the $C 2 / c$ structure that is a local energy minimum of 20-atom cell $\mathrm{BiFeO}_{3}$ (left) and the Pbam structure that is a global energy minimum of bulk $\mathrm{PbZrO}_{3}$ (right). Each image shows the distribution of $\mathrm{O}_{6}$ octahedra in each plane perpendicular to the inequivalent axis of each structure, together with arrows that represent the displacement of $\mathrm{Bi}$ atoms in the nearest parallel plane to those octahedra.

possible, and the conventional cell of the $C 2 / c$ phase for the other cases. The existence of negative eigenvalues of the dynamical matrix was checked for those extended 40-atom unit cells (and not only for the cell in which the minima was found, as in Table II). If soft modes exist, we give the energy of the structure in brackets. Thus, for example, structures 3,11 , and 16 are reported as local minima in Table II because they are so in their respective 20-, 10-, and 20-atom unit cells where they were found by USPEX; however, when we used 40-atom unit cells soft modes appeared in those three cases. The structure that results when we follow the soft mode of the $C 2 / c$ phase is the subject of next subsection.

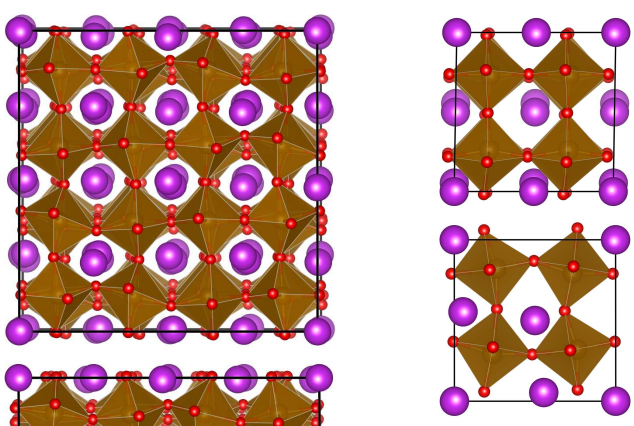

(b) $P c$
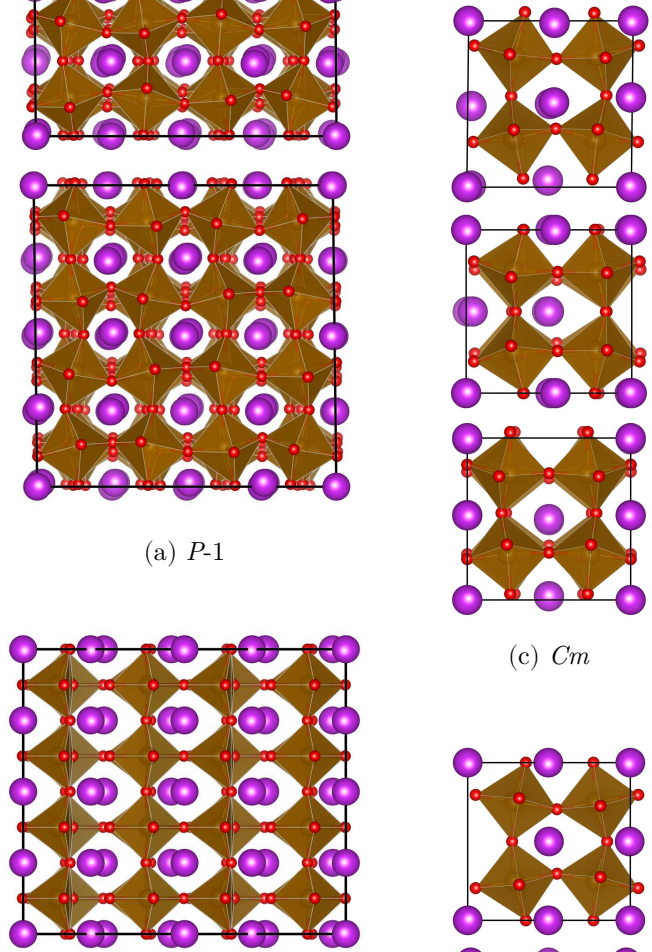

(c) $\mathrm{Cm}$
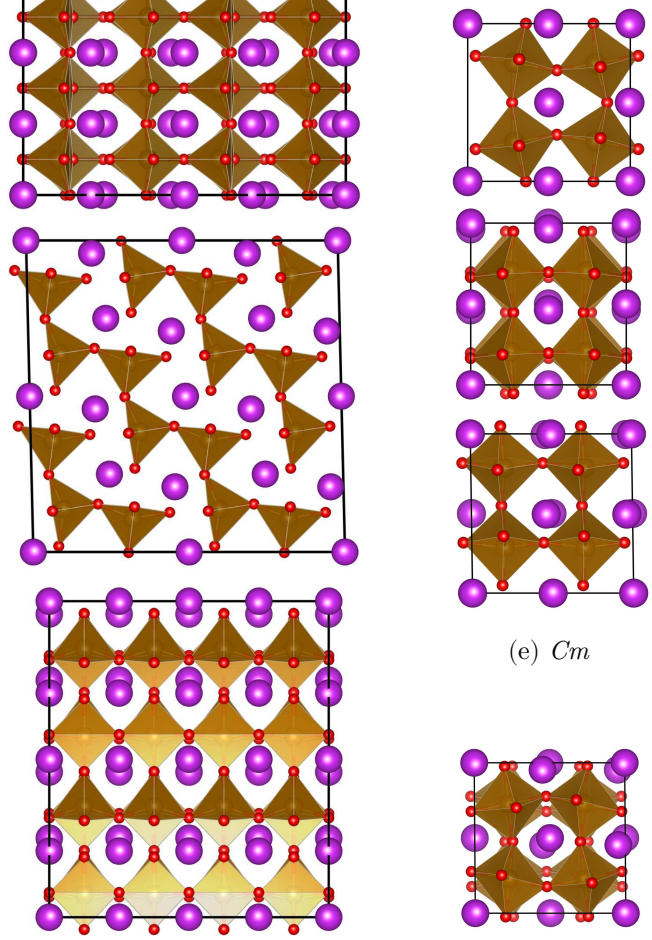

(d) $P m c 2$

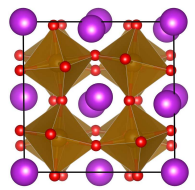

(f) $R-3$

FIG. 3. Low energy structures of $\mathrm{BiFeO}_{3}$ displaying G-type antiferromagnetism. 
TABLE III. Characteristics of the structures found after our search over 10-atom and 20-atom unit cells of five Bi-based transition-metal perovskite oxides. For each material there are four columns: S.G. contains the space group of the structure; M. contains the lowest energy magnetic configuration (one capital letter indicates antiferromagnetic type, FM indicates ferromagnetism - an asterisk is added if there is charge ordering); $\Delta E$ is the difference in energy (in meV/f.u.) with the structure that is the accepted experimental ground state; and $c / a$ is the ratio between the most dissimilar lattice parameter and the average of the other two (in the perovskite pseudocubic unit cell). When the energy value is underlined, it means that this structure was found by the USPEX code; otherwise, it was found by adapting a structure found by USPEX in one of the other materials. When the energy value is in brackets, it means that this is not a local minimum of the energy in the corresponding 40-atom pseudocubic cell, but a saddle point. Structures in the same line are considered to be the same (they show the same main distortions in terms of cation displacements and $\mathrm{O}_{6}$ rotations), even if in some cases Jahn-Teller distortions cause the space group to vary.

\begin{tabular}{|c|c|c|c|c|c|c|c|c|c|c|c|c|c|c|c|c|c|c|c|}
\hline \multicolumn{4}{|c|}{$\mathrm{BiScO}_{3}$} & \multicolumn{4}{|c|}{$\mathrm{BiCrO}_{3}$} & \multicolumn{4}{|c|}{$\mathrm{BiMnO}_{3}$} & \multicolumn{4}{|c|}{$\mathrm{BiFeO}_{3}$} & \multicolumn{4}{|c|}{$\mathrm{BiCoO}_{3}$} \\
\hline S.G. & M. & $\Delta E$ & $c / a$ & S.G. & M. & $\Delta E$ & $c / a$ & S.G. & M. & $\Delta E$ & $c / a$ & S.G. & M. & $\Delta E$ & $c / a$ & S.G. & M. & $\Delta E$ & $c / a$ \\
\hline$P 4 m m$ & 0 & (409) & 1.296 & & & & & $P 4 m m$ & $\mathrm{~A}$ & (390) & 1.264 & $P 4 m m$ & $\mathrm{C}$ & (140) & 1.282 & $P 4 m m$ & $\mathrm{C}$ & $\underline{0}$ & 1.257 \\
\hline$R 3 c$ & 0 & $\underline{-45}$ & 1.000 & $R 3 c$ & $\mathrm{G}$ & $\underline{-22}$ & 1.000 & $C c$ & FM & $\underline{7}$ & 0.975 & $R 3 c$ & $\mathrm{G}$ & $\underline{0}$ & 1.000 & $C c$ & $\mathrm{G}$ & $\underline{38}$ & 1.026 \\
\hline \multirow[t]{3}{*}{ Pnma } & 0 & $\underline{-35}$ & 0.994 & Pnma & $\mathrm{G}$ & -59 & 0.996 & Pnma & FM & -17 & 0.962 & Pnma & $\mathrm{G}$ & $\underline{28}$ & 0.992 & Pnma & $\mathrm{G}$ & 47 & 1.014 \\
\hline & & & & & & & & $C 2 / c$ & $\mathrm{FM}^{*}$ & $\underline{-15}$ & 0.985 & & & & & & & & \\
\hline & & & & & & & & $P 2_{1} / c$ & $\mathrm{FM}^{*}$ & $\underline{-9}$ & 0.990 & & & & & & & & \\
\hline \multirow[t]{3}{*}{$C 2 / c$} & 0 & 0 & 0.984 & $C 2 / c$ & $\mathrm{G}$ & (0) & 0.993 & $C 2 / c$ & FM & $(0)$ & 1.017 & $C 2 / c$ & $\mathrm{G}$ & $(\underline{68})$ & 0.988 & $C 2 / c$ & $\mathrm{G}$ & 53 & 0.979 \\
\hline & & & & & & & & $R 3$ & $\mathrm{FM}^{*}$ & $\underline{2}$ & 1.000 & & & & & & & & \\
\hline & & & & & & & & $R \overline{3}$ & $\mathrm{FM}^{*}$ & $\underline{20}$ & 1.000 & & & & & & & & \\
\hline Pna $2_{1}$ & 0 & -25 & 1.009 & & & & & & & & & & & & & & & & \\
\hline$P \overline{1}$ & 0 & 28 & 1.011 & $P \overline{1}$ & $\mathrm{G}$ & (26) & 0.994 & $P \overline{1}$ & FM & $(37)$ & 1.019 & $P \overline{1}$ & $\mathrm{G}$ & $\underline{93}$ & 1.012 & $P \overline{1}$ & $\mathrm{G}$ & 88 & 0.964 \\
\hline$R \overline{3}$ & 0 & $\underline{127}$ & 1.000 & $R \overline{3}$ & $\mathrm{G}$ & 43 & 1.000 & $P \overline{1}$ & FM & 64 & 0.952 & $R \overline{3}$ & G & $\underline{164}$ & 1.000 & $P \overline{1}$ & $\mathrm{G}$ & $\underline{147}$ & 1.023 \\
\hline \multirow[t]{7}{*}{$\mathrm{Cm}$} & 0 & $\underline{63}$ & 0.988 & $\mathrm{Cm}$ & $\mathrm{G}$ & (95) & 1.003 & $\mathrm{Cm}$ & FM & 97 & 0.949 & $\mathrm{Cm}$ & G & $\underline{111}$ & 1.015 & $\mathrm{Cm}$ & G & (139) & 1.025 \\
\hline & & & & & & & & & & & & $C c$ & $\mathrm{C}$ & $\underline{97}$ & 1.255 & & & & \\
\hline & & & & & & & & $P n a 2_{1}$ & A & 139 & 1.149 & Pna $2_{1}$ & $\mathrm{C}$ & $\underline{100}$ & 1.257 & & & & \\
\hline & & & & & & & & & & & & $\mathrm{Cm}$ & $\mathrm{C}$ & $\underline{103}$ & 1.273 & & & & \\
\hline & & & & & & & & & & & & $C c$ & $\mathrm{C}$ & $\underline{105}$ & 1.264 & & & & \\
\hline & & & & & & & & $P c$ & $\mathrm{~A}$ & 212 & 1.220 & $P c$ & $\mathrm{C}$ & $\underline{106}$ & 1.265 & & & & \\
\hline & & & & & & & & & & & & $P m n 2_{1}$ & $\mathrm{C}$ & $\underline{106}$ & 1.272 & & & & \\
\hline$C c$ & 0 & $\underline{61}$ & 1.022 & & & & & & & & & & & & & $C c$ & G & 107 & 1.039 \\
\hline \multirow[t]{2}{*}{$P c$} & 0 & $\underline{63}$ & 0.991 & & & & & & & & & & & & & & & & \\
\hline & & & & & & & & & & & & $\mathrm{Cm}$ & $\mathrm{C}$ & $(\underline{109})$ & 1.272 & & & & \\
\hline$P c$ & 0 & 69 & 1.004 & $P c$ & $\mathrm{G}$ & $(122)$ & 1.015 & $P c$ & FM & (127) & 1.000 & $P c$ & $\mathrm{G}$ & $\underline{110}$ & 0.996 & $P c$ & $\mathrm{G}$ & (148) & 1.025 \\
\hline$P n a 2_{1}$ & 0 & $\underline{64}$ & 0.997 & $P n a 2_{1}$ & $\mathrm{G}$ & $\underline{125}$ & 1.001 & $P n a 2_{1}$ & FM & 122 & 0.990 & $P n a 2_{1}$ & $\mathrm{G}$ & 126 & 0.992 & $P n a 2_{1}$ & $\mathrm{G}$ & 136 & 1.020 \\
\hline Ama2 & 0 & $(\underline{65})$ & 0.996 & & & & & Ama2 & FM & (131) & 0.988 & & & & & & & & \\
\hline$P m c 2_{1}$ & 0 & (208) & 0.879 & & & & & $P m c 2_{1}$ & FM & (146) & 0.907 & $P m c 2_{1}$ & $\mathrm{G}$ & $\underline{119}$ & 0.885 & & & & \\
\hline \multirow[t]{2}{*}{$P m c 2_{1}$} & 0 & $(236)$ & 0.970 & $P m c 2_{1}$ & $\mathrm{G}$ & (169) & 1.014 & & & & & $P m c 2_{1}$ & $\mathrm{G}$ & (163) & 0.969 & $P m c 2_{1}$ & G & $(\underline{111})$ & 1.014 \\
\hline & & & & & & & & & & & & $F m m 2$ & $\mathrm{C}$ & $\underline{116}$ & 1.247 & & & & \\
\hline \multirow[t]{2}{*}{$\mathrm{Cm}$} & 0 & $(81)$ & 0.968 & $\mathrm{Cm}$ & $\mathrm{G}$ & (118) & 1.024 & $\mathrm{Cm}$ & FM & $(121)$ & 1.039 & $\mathrm{Cm}$ & $\mathrm{G}$ & (125) & 0.966 & $\mathrm{Cm}$ & G & (117) & 1.031 \\
\hline & & & & & & & & & & & & & & & & $C c$ & $\mathrm{G}$ & $\underline{144}$ & 1.041 \\
\hline $\mathrm{Cm}$ & 0 & $\underline{102}$ & 1.009 & & & & & & & & & & & & & & & & \\
\hline
\end{tabular}

Another example will help to clarify how information is reported in Table III. For $\mathrm{BiCoO}_{3}$, UsPEx found the supertetragonal $P 4 \mathrm{~mm}$ phase that is indeed the known experimental ground state of the material; so an underlined value of $0 \mathrm{meV} / \mathrm{fu}$ is added to Table III in the $\mathrm{BiCoO}_{3}$ column. When computing energies of different magnetic arrangements, antiferromagnetism of the $\mathrm{C}$ type is favored (also in agreement with experiment), so this is too reflected, together with the large $c / a$ ratio. We then run the same structure for the other four materials (we started optimizations by simply changing the $B$ cation and using a initial cell volume adjusted proportionally to the size of the new cation). In three cases $\left(\mathrm{BiScO}_{3}\right.$, $\mathrm{BiMnO}_{3}$, and $\mathrm{BiFeO}_{3}$ ) the optimization converged, but there were negative dynamical matrix eigenvalues in the $2 \times 2 \times 2$ pseudocubic cell, so the energy values are in 
brackets. In the other case, $\mathrm{BiCrO}_{3}$, the supertetragonal $P 4 \mathrm{~mm}$ phase would just not be a special point of the energy surface.

Once again, we see a richness of polymorphs that is unique to bismuth perovskite oxides. The phases here include the previously reported ones for these five materials, most notably their ground state form according to experiment and the Pnma phase that they all reach at high enough temperature or pressure. We can establish three groups of structures: phases with similar pseudocubic lattice constants, phases with one pseudocubic lattice constant significantly larger than the other two (supertetragonal phases), and phases with one pseudocubic lattice constant significantly smaller than the other two (inverse supertetragonal phases). Among the first group, a phase that appears for all five materials as a local minimum is the $R 3 \mathrm{c}$ phase (global minimum of $\mathrm{BiFeO}_{3}$ ). In $\mathrm{BiMnO}_{3}$ and $\mathrm{BiCoO}_{3}$ this phase is slightly distorted because $\mathrm{Mn}^{+3}$ and $\mathrm{Co}^{+3}$ are Jahn-Teller active ions, so their space group becomes actually $C c$; however, the main distortions in the structures are still the large cation displacement along the (111) pseudocubic direction and a $\mathrm{O}_{6}$ rotation similar to $a^{-} a^{-} a^{-}$, so we group these minima in the same line of the Table. The supertetragonal polar phases appear only in $\mathrm{BiMnO}_{3}$, $\mathrm{BiFeO}_{3}$ and $\mathrm{BiCoO}_{3}$, with $\mathrm{A}$-antiferromagnetic ordering $\left(\mathrm{BiMnO}_{3}\right)$ and $\mathrm{C}$-antiferromagnetic ordering $\left(\mathrm{BiFeO}_{3}\right.$ and $\left.\mathrm{BiCoO}_{3}\right)$. On the other hand, non-supertetragonal polar and non-polar phases can be seen in all five materials; they favour G-antiferromagnetic ordering in $\mathrm{BiCrO}_{3}$, $\mathrm{BiFeO}_{3}$, and $\mathrm{BiCoO}_{3}$, while ferromagnetic ordering and A-antiferromagnetic ordering are very close in $\mathrm{BiMnO}_{3}$, as previously reported ${ }^{[20}$ The inverse supertetragonal phases are analyzed in more detail in subsection E.

Apart from the high number of structures listed, a few other facts deserve to be commented in relation to TableIII. First, there are several instances of negative energies for $\mathrm{BiScO}_{3}, \mathrm{BiCrO}_{3}$, and $\mathrm{BiMnO}_{3}$. This implies that according to our calculations, those phases have energies below that of the assumed experimental ground state. In a previous article we reported that for $\mathrm{BiMnO}_{3} \mathrm{DFT}+U$ does not agree with experiment in that the $C 2 / c$ phase is the one with the lowest energy, but that when using hybrid methods reconciliation with experiment is achieved. Now we see that the situation is extended to $\mathrm{BiScO}_{3}$ and $\mathrm{BiCrO}_{3}$. In next subsection we look in more depth at this issue. Second, we have found that $\mathrm{BiMnO}_{3}$ shows some unique phases where charge separation is present$\mathrm{Mn}^{+2}$ and $\mathrm{Mn}^{+4}$ ions coexist. This is further discussed in subsection D.

As a point of comparison, we also run analogous USPEX optimization calculations for prototypical perovskites $\mathrm{BaTiO}_{3}$ and $\mathrm{PbTiO}_{3}$. Full runs as those described in the Methods section produced just the known ground states of these materials: the rhombohedral $R 3 \mathrm{~m}$ phase for $\mathrm{BaTiO}_{3}$ and the tetragonal $P 4 m m$ phase for $\mathrm{PbTiO}_{3}$. No other local minima was found in 20-atom unit cells (the tetragonal $P 4 m m$ and orthorhombic Amm2 phases

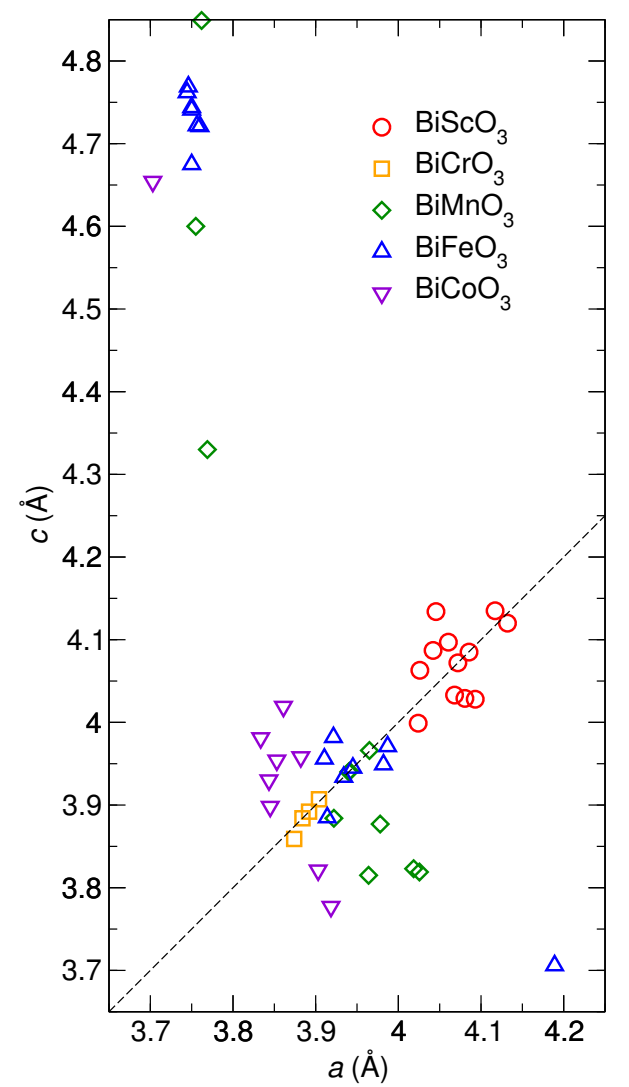

FIG. 4. Out-of-plane lattice parameter $c$ versus in-plane lattice parameter $a$ (the average of the two closest lattice parameters in the pseudocubic setting), for the structures listed in Table III that are local minima of the energy. The discontinuous line corresponds to $c=a$.

of $\mathrm{BaTiO}_{3}$ are saddle points, not minima).

To give a graphical idea of the richness of $\mathrm{BiXO} \mathrm{O}_{3}$ phases found, Fig. 4 shows the value of the $c$ lattice parameter versus the average $a$ and $b$ parameters for those phases of Table III that are local minima. The lattice parameters are sorted so that $a$ and $b$ are as close as possible to each other; we do this with the idea in mind that a possible way to access local minima of these materials is to grow them as epitaxial films on square perovskite lattices - then, the in-plane lattice parameter would likely be around $(a+b) / 2$ and the out-of-plane lattice parameter would be around $c$. It is apparent from the graph that one cluster of supertetragonal phases appear at the top left of the figure, while most of the rest of the phases have $c / a$ ratios not far from one. The outlier at the bottom right part of the graph is phase 15 of $\mathrm{BiFeO}_{3}$ (in this graph only phases that are minima in the 40-atom unit cells described earlier are represented, so the inverse supertetragonal phases of other materials are absent).

This adaptability of the $\mathrm{Bi}$ ion to different environments of perovskite variations implies not only that phases other than the ground state might be stabi- 
lized by strain or pressure, but also that these materials should have richer surfaces and interfaces than typical perovskites. For example, some $\mathrm{BiFeO}_{3}$ domain walls can be seen as narrow regions in which the structure corresponds to a diffferent polymorph ${ }^{26 / 51}$ and the same it is true for its surfaces. 53

\section{Ground State of $\mathrm{BiScO}_{3}, \mathrm{BiCrO}_{3}$, and $\mathrm{BiMnO}_{3}$}

We move on now to the issue of the phases of $\mathrm{BiScO}_{3}$, $\mathrm{BiCrO}_{3}$, and $\mathrm{BiMnO}_{3}$ that show lower energies than the one of the experimental ground state of these materials, the phase with $C 2 / c$ symmetry. In our previous study of $\mathrm{BiMnO}_{3}{ }^{20}$ we reported that $\mathrm{DFT}+U$ calculations very similar to the ones presented here indeed stablish that the Pnma phase has a somewhat lower energy than the $C 2 / c$ phase. We also showed that when using hybrid functionals, this ordering is reversed, and the $C 2 / c$ phase becomes the lowest-energy one, in accordance to the latest experiments about the structure of this material. $\frac{10}{10}$ Now we see that for $\mathrm{BiScO}_{3}$ and $\mathrm{BiCrO}_{3}$ we also obtain that the Pnma phase has lower energy than the $C 2 / c$ phase. Moreover, in this case this is also true for the $R 3 c$ phase.

More puzzingly, the data in Table III tells that the $C 2 / c$ phase of $\mathrm{BiCrO}_{3}$ and $\mathrm{BiMnO}_{3}$ shows a soft mode when the conventional 40-atom unit cell is used. When we perturb slightly the atomic positions of these structures by following the eigenvector of the soft mode, indeed the energy of $\mathrm{BiCrO}_{3}$ and $\mathrm{BiMnO}_{3}$ (and also $\mathrm{BiFeO}_{3}$ ) goes down, while this does not happen in $\mathrm{BiScO}_{3}$ (and $\mathrm{BiCoO}_{3}$ ). This is consistent with the structure being a minimum in $\mathrm{BiScO}_{3}$ (and $\mathrm{BiCoO}_{3}$ ), but a saddle point in $\mathrm{BiCrO}_{3}$ and $\mathrm{BiMnO}_{3}$ (and $\mathrm{BiFeO}_{3}$ ). What is the structure that appears if we keep following the eigenvector of the soft mode and then let the atoms relax? This structure has 40 atoms in its unit cell (and therefore could not have been found in our USPEX search over 10-atom and 20-atom cells), and space group $P 2_{1} / c$. In addition to the distortions quoted in Table II for the $C 2 / c$ phase, this $P 2_{1} / c$ phase has two other prominent ones: an antipolar Bi displacement along the (001) pseudocubic axis, and a $\left(a^{0} a^{0} c^{+}\right) \mathrm{O}_{6}$ rotation. In all, it shows a similar $\mathrm{O}_{6}$ rotation pattern to that of the Pnma phase, and a complicated antipolar Bi pattern associated mainly to the $(2 \pi / a)(1 / 4,1 / 4,1 / 4)$ point of the simple cubic first Brillouin zone.

Figure 5 (left panels) shows the energy as we interpolate linearly the atomic positions and lattice vectors from the $C 2 / c$ phase to the $P 2_{1} / c$ phase, for all five Bibased perovskites. It is apparent that the curvature at the $C 2 / c$ point is much larger for those materials where this phase is a minimum $\left(\mathrm{BiScO}_{3}\right.$ and $\left.\mathrm{BiCoO}_{3}\right)$ than for the others. When the $C 2 / c$ phase is a saddle point of the energy surface, then the $P 2_{1} / c$ phase corresponds to a minimum with lower energy; when instead the $C 2 / c$ phase is a minimum, then the $P 2_{1} / c$ phase is also a min-

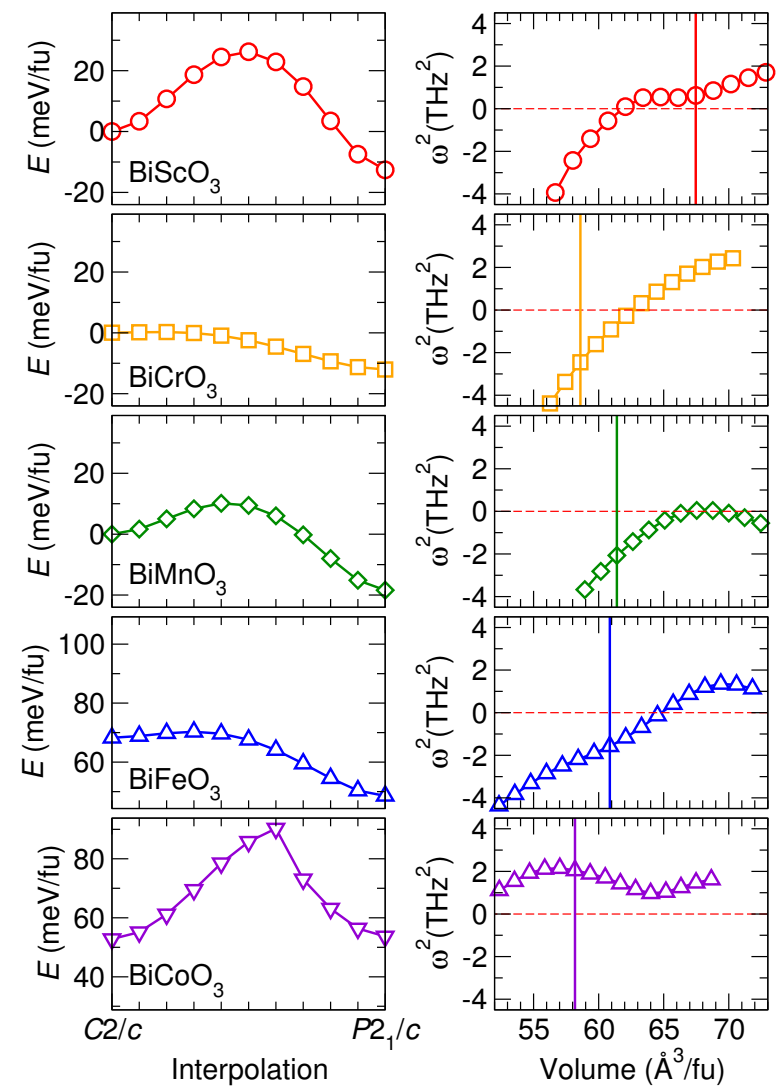

FIG. 5. Left panels: energy of the structures that result from a linear interpolation of atomic positions and lattice vectors between the $C 2 / c$ phase and the $P 2_{1} / c$ phases of the materials quoted. Right panels: square of the frequency of the mode that connects the $C 2 / c$ and $P 2_{1} / c$ phases as a function of the volume of the $C 2 / c$ unit cell; the vertical lines mark the volume of the cell with optimal lattice parameters.

imum, but it might exist at a lower energy $\left(\mathrm{BiScO}_{3}\right)$ or at a higher one $\left(\mathrm{BiCoO}_{3}\right)$.

What is the reason for the different character of the $C 2 / c$ phases in the different oxides analyzed here? To help us answer this question, Figure 5 (right panels) contains the value of the frequency of the mode that becomes soft in $\mathrm{BiCrO}_{3}$ and $\mathrm{BiMnO}_{3}$, for different values of unit cell volume - i.e., we increased the length of the $a, b$, and $c$ lattice parameter of the $C 2 / c$ conventional unit cell by some amount, relaxed the atoms with those fixed lattice vectors, computed the eigenvalues of the dynamical matrix, and plotted the square of the eigenvalue of interest in the graphs. What results is consistent with a similar behavior of this frequency for all five materials: at large values of volume there is room for the $\mathrm{O}_{6}$ cages without the need for the extra rotation of the $P 2_{1} / c$ phase, so the eigenvalues are positive, while at small enough volumes this is not the case and the structure adds those rotations as a way to avoid compressing the bonds between transition metals and oxygens further. In between those regimes, the rotations and the Bi displacements compete, giving rise to the non-monotonic behaviour seen in the 
TABLE IV. Results of HSE06 calculations of the most stable structures of bulk $\mathrm{BiScO}_{3}, \mathrm{BiCrO}_{3}$, and $\mathrm{BiMnO}_{3}$. For each material we report its lowest magnetic ordering, the energy difference with the $C 2 / c$ phase (in $\mathrm{meV} /$ f.u.), and its band gap (in eV).

\begin{tabular}{rccccccccc}
\hline \hline \multicolumn{1}{c}{$\mathrm{BiScO}_{3}$} & \multicolumn{3}{c}{$\mathrm{BiCrO}_{3}$} & \multicolumn{3}{c}{$\mathrm{BiMnO}_{3}$} \\
Phase & M. & $\Delta E$ & Gap & M. & $\Delta E$ & Gap & M. & $\Delta E$ & Gap \\
\hline$C 2 / c$ & 0 & 0 & 4.3 & G & 0 & 3.1 & FM & 0 & 1.8 \\
$P 2_{1} / c$ & 0 & 14 & 4.0 & G & -2 & 3.1 & FM & 1 & 1.9 \\
$R 3 c$ or $C c$ & 0 & 4 & 4.2 & G & -13 & 3.3 & A & 33 & 2.5 \\
$P n m a$ & 0 & 64 & 4.0 & G & -8 & 2.7 & FM & 53 & 1.7 \\
\hline \hline
\end{tabular}

panels. Depending on what the optimal value of the volume is for a given material, different parts of the curve are accessed, and the different behaviours listed earlier result.

The previous discussion points out that the relative ordering of the $C 2 / c$ and $P 2_{1} / c$ phases is a subtle effect. To further investigate this issue, and the one of the negative energies of Table III, we have used hybrid functionals. These computationally demanding calculations represent today one of the most accurate types of firstprinciples calculations that can be performed to compute

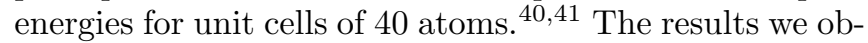
tained are quoted in Table IV. Some of the $\mathrm{BiMnO}_{3}$ data had been reported before ${ }^{20}$ showing that the $C 2 / c$ phase was lower in energy than the $R 3 c$ and Pnma phases; now we see that the $P 2_{1} / c$ phase has very close energy to the $C 2 / c$ phase, but $C 2 / c$ is still the ground state according to hybrid calculations. Also in $\mathrm{BiScO}_{3}$ the $C 2 / c$ phase is the ground state according to our HSE06 calculations, in agreement with experiment. For $\mathrm{BiCrO}_{3}$, however, the hybrid calculations put the analyzed structures in a small bracket of energies where the $C 2 / c$ phase is not the lowest-energy one (whether this is an artifact or not of the calculations is difficult to assess, given the small differences in energy involved). Computing possible phonon instabilities of these phases with the HSE06 hybrid functional would require ten to twenty times more computer power than the calculations reported in Table IV, which are already computationally demanding. Instead, the results reported in Fig. 5 give evidence that when the $C 2 / c$ phase is lower in energy than the $P 2_{1} / c$ phase both correspond to energy minima. When using hybrids we see that the $C 2 / c$ phase in $\mathrm{BiScO}_{3}$ and $\mathrm{BiMnO}_{3}$ is lower in energy than the $P 2_{1} / c$ phase, so we expect both structures to be minima ( $C 2 / c$ being the global one, in agreement with experiment).

\section{Charge-Ordering Phases in $\mathrm{BiMnO}_{3}$}

Another interesting feature reported in Table III is the presence of four phases of $\mathrm{BiMnO}_{3}$ that possess charge
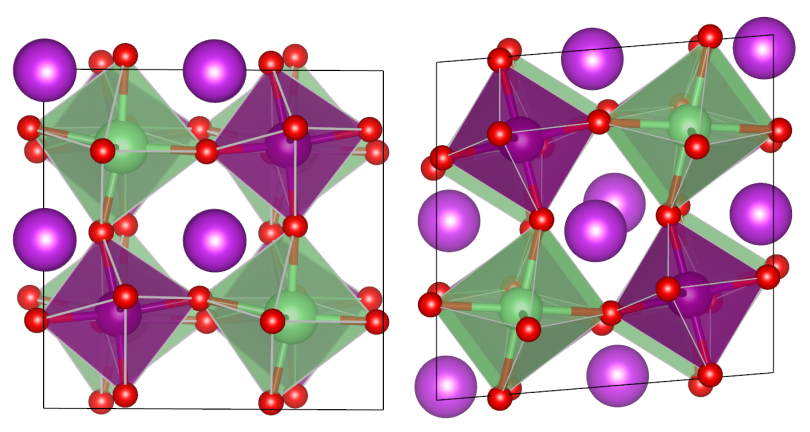

FIG. 6. 40-atom unit cells for two of the charge-ordering phases of $\mathrm{BiMnO}_{3}$ found in this work: a $R 3 c$-like phase (left) similar to the ground state of $\mathrm{BiFeO}_{3}$ but with a rock-salt pattern imposed on the perovksite $B$ sites, and a Pnma-like phase (right) similar to the high-pressure phase of these materials but with the same rock-salt pattern. The larger (smaller) octahedra enclose $\mathrm{Mn}^{+2}\left(\mathrm{Mn}^{+4}\right)$ ions.

ordering. These correspond, in order of increasing energy, to variations of the $C 2 / c, P n m a, R 3 c$, and $R \overline{3}$ phases, where a rocksalt pattern of $\mathrm{Mn}^{2+}$ and $\mathrm{Mn}^{4+}$ ions exists in the $B$ site of the perovksite (unit cells of two of the phases found are pictured in Fig. 6). Charge-ordering phases of perovskites have been reported experimentally before, with the ordering taking place either in the $B$ site (e.g., $\mathrm{Mn}^{+3}$ and $\mathrm{Mn}^{+4}$ in $\mathrm{Ln}_{1-x} A_{x} \mathrm{MnO}_{3}$, where $\mathrm{Ln}=$ rare earth and $A=\mathrm{Ca}, \mathrm{Sr}^{\sqrt[54]{4}}$ ) or in the $A$ site (e.g., $\mathrm{Bi}^{+3}$ and $\mathrm{Bi}^{5}$ in $\mathrm{BiNiO}_{3} \sqrt{55}$ ). However, no $A B \mathrm{O}_{3}$ perovskite with charge ordering in $B$ seems to have been found so far.

To check how robust the prediction done with $\mathrm{DFT}+U$ is, we also carried out hybrid calculations for these phases (assuming that the spins are oriented parallel to each other). Table V] contains the results of a comparison of the structure of these variations and the one of the regular phases. In particular, we show the average Mn$\mathrm{O}$ distance obtained for each individual $\mathrm{Mn}$ ion of the structure; these results agree well with the picture of ionic bonding that emerges from the radii given by Shannon $\sqrt{57}$ $\left(1.35 \AA\right.$ for $\mathrm{O}^{-2}, 0.53 \AA$ for $\mathrm{Mn}^{+4}, 0.65 \AA$ for $\mathrm{Mn}^{+3}$, $0.82 \AA$ for $\mathrm{Mn}^{+2}$ ).

As seen in Table $V$ the energy differences between the phases with charge ordering and the ground state are larger than the ones reported in Table III using DFT $+U$. Still, these differences are similar to the ones between experimentally grown supertetragonal phases of $\mathrm{BiFeO}_{3}$ and its ground state. It might therefore be feasible to stabilize some of these phases; a possible route could be to grown $\mathrm{BiMnO}_{3}$ epitaxially on a perovskite substrate that shows a checkerboard pattern compatible with the distortions, e.g., using as substrate one of the many ordered double perovskites that are known. $\underline{58}$ 
TABLE V. Results of HSE06 calculations for some of the regular phases of $\mathrm{BiMnO}_{3}$, and for its charge-ordering phases. For each material we report the energy difference of the given phase (parallel spins) with respect to the $C 2 / c$ phase (in $\mathrm{meV} /$ f.u.), the lattice parameters, and the average Mn-O distances.

\begin{tabular}{|c|c|c|c|c|c|c|}
\hline Phase $\Delta E$ & $\begin{array}{r}\text { Lat } \\
a(\AA)\end{array}$ & $\begin{array}{l}\text { t. para } \\
b(\AA)\end{array}$ & $\begin{array}{l}\mathrm{am} . \\
c(\AA)\end{array}$ & $\begin{array}{r}\mathrm{Mn}- \\
\mathrm{Mn}^{4+}\end{array}$ & $\begin{array}{l}\mathrm{O} \text { dist. } \\
\mathrm{Mn}^{3+}\end{array}$ & $\begin{array}{c}(\AA) \\
\mathrm{Mn}^{2+}\end{array}$ \\
\hline \multicolumn{7}{|c|}{ Regular phases: } \\
\hline$C 2 / c \quad 0$ & 3.903 & 3.903 & 3.975 & - & 2.02 & - \\
\hline$R 3 c$-like 36 & 4.026 & 4.026 & 3.772 & - & 2.02 & - \\
\hline Pnma 53 & 3.949 & 3.949 & 3.764 & - & 2.03 & - \\
\hline$R \overline{3}$-like 102 & 3.986 & 4.072 & 3.749 & - & 2.02 & - \\
\hline \multicolumn{7}{|c|}{ Phases with charge ordering: } \\
\hline$C 2 / c$-like 97 & 3.929 & 3.929 & 3.857 & 1.91 & - & 2.13 \\
\hline$R 3 c$-like 152 & 3.919 & 3.919 & 3.919 & 1.92 & - & 2.09 \\
\hline Pnma-like 160 & 3.923 & 3.923 & 3.870 & 1.92 & - & 2.12 \\
\hline$R \overline{3}$-like 145 & 3.918 & 3.918 & 3.918 & 1.91 & - & 2.13 \\
\hline
\end{tabular}

\section{E. Highly Strained Epitaxial Film Phase}

In Fig. 4 we plotted the behaviour of the lattice parameter that most differ from the other two $(c)$ as a function of the average of those other two $(a)$. This is to connect with the topic of epitaxial films that are grown on a square substrate, a common situation for perovskites grown on perovskites or on other materials of cubic symmetry - the bulk forms are likely to adapt more easily to the substrate in the orientation that matches best two of its lattice parameters to the substrate square parameter. The points in that figure can be divided in three groups. In most cases, $c / a$ is between 0.9 and 1.1, and we find there typical phases of perovskites, such as the $R 3 c, C 2 / c$, and Pnma ones. When $c / a$ is larger than 1.1 we obtain the supertetragonal phases similar to the ground state of $\mathrm{BiCoO}_{3}$ that we have already mentioned. There is a third set of points, with $c / a$ below 0.9 , corresponding to an orthorhombic $P m c 2_{1}$ phase first reported by Yang et al. ${ }^{[50}$ after they found computationally as favorable epitaxial phase in the region of large tensile strains of $\mathrm{BiFeO}_{3}$ and $\mathrm{PbTiO}_{3}$ (they quote a strain of $5 \%)$.

Now we show that this phase is a local minimum of the bulk of $\mathrm{BiFeO}_{3}$, and a saddle point in some of the related materials. This is relevant because it implies that, like for its supertetragonal phase, $\mathrm{BiFeO}_{3}$ can stand nominally large epitaxial strains without creating a myriad of defects - the material grows into a phase whose lattice parameters match very well those of the substrate, and therefore very small stresses are present in the film.

There is an interesting detail in Table III related to this inverse supertetragonal phase (initially found in our $\mathrm{BiFeO}_{3}$ USPEX search): another phase with the same set of main distortions and the same $P m c 2_{1}$ space group
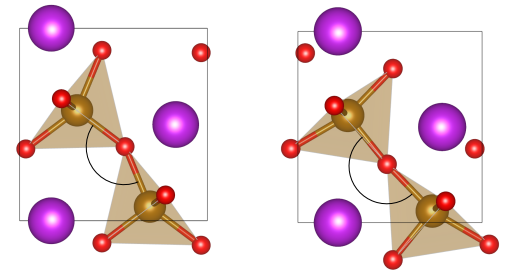

(a)

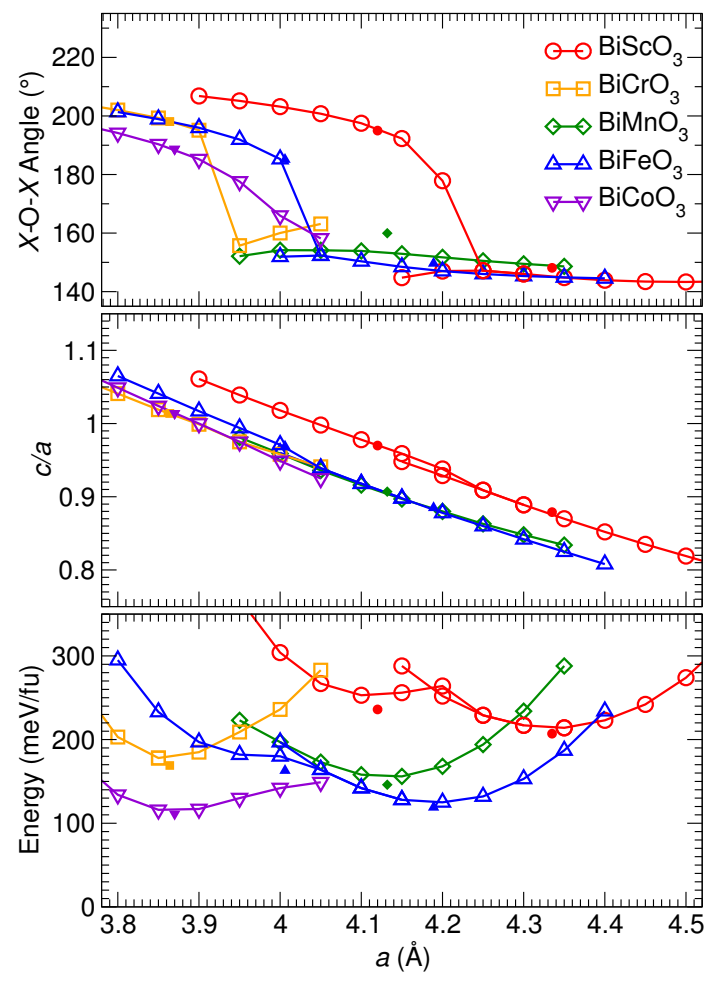

(b)

FIG. 7. (a) Primitive 10-atom unit cell of the inverse supertetragonal phase of $P m c 2_{1}$ symmetry with $c / a$ ratio much smaller than 1 (left), and of the related phase that has $c / a$ around 1 (right); the $X-\mathrm{O}-X$ angle that distinguishes these phases is highlighted. (b) Behavior of this $X-\mathrm{O}-X$ angle, $c / a$, and energy relative to the ground state for films of those two phases when grown epitaxially on a square substrate of given in-plane lattice parameter $a$; the small solid symbols represent bulk properties of the phases labelled as $P m c 2_{1}$ in Table III.

exists, but with a $c / a$ ratio much closer to 1 . This similar phase appeared in the USPEX search of $\mathrm{BiCoO}_{3}(111$ $\mathrm{eV} /$ fu above the $P 4 \mathrm{~mm}$ ground state). Apart from $c / a$, the difference between the two phases is that the $\left(a^{0} a^{0} c^{+}\right)$ rotation occurs in opposite directions with respect to the mix of polar and antipolar Bi displacements, as shown in Fig. 7 (a). As seen in the Table, both phases are present in $\mathrm{BiFeO}_{3}$ and $\mathrm{BiScO}_{3}$, but we have only confirmed one of them for the other three materials.

With the goal of further understanding these phases and of assesing their suitability as possible ground states in epitaxial films at large tensile strains, Fig. 7(b) shows 
the results of constraining them to square substrates. In particular, the bottom graph shows that it costs little energy to adapt the bulk phases to the elastic constraint imposed by the substrate (the energy difference between the minimum of the open symbols curves and the small filled symbols is very small). It also reveals that these two isosymmetric structures correspond to two actual different phases, as it can be seen more clearly for the cases of $\mathrm{BiFeO}_{3}$ and $\mathrm{BiScO}_{3}$, where at intermediate values of $a$ the two types can be optimized. The middle graph shows how the $c / a$ ratio is almost linear with $a$, and almost identical for all cases, but when the larger $\mathrm{Sc}^{+3}$ ion is present. The top graph gives the clearest evidence of the values of $a$ for which one or the other phase is the favored one. Finally, the energy curves help to understand why in some cases our bulk calculations find only one of the two phases: they are very close in the search space of crystal configurations, and when free bulk optimizations are performed these end up in the lowest special point available - the inverse supertetragonal one $\left(\mathrm{BiMnO}_{3}\right)$ or its companion $\left(\mathrm{BiCoO}_{3}\right.$ and $\left.\mathrm{BiCrO}_{3}\right)$. For $\mathrm{BiFeO}_{3}$ and $\mathrm{BiScO}_{3}$ the two phases are further away from each other, and optimizations can access either of them.

In all, our calculations related to this inverse supertetragonal phase reinforce the point made by Yang et al .50 that materials such as $\mathrm{BiFeO}_{3}$ (and perhaps other Bibased perovskites if they can be stabilized) might exist in epitaxial polymorphs with a large in-plane polarization, and with other interesting properties that arise due to the special network of square pyramids present.

\section{F. Band Gaps}

In recent times, the search for clean energy has spurred the optimization of materials for converting solar light into electricity. Optimal materials have bandgaps that are smaller than those typical of ferroelectric perovskite oxides, but efforts have been made to reduce those bandgaps, and in this way couple the ferroelectric functionalities to those of an energy material. ${ }^{[5 \mid 60}$ This motivated us to analyze the bandgaps predicted for the phases identified in our search.

Experimentally, the optical bandgap of $\mathrm{BiFeO}_{3}$ has been measured by several groups, with results ranging from 2 to $3 \mathrm{eV} !^{\frac{2}{2}}$ For the other Bi-based materials of this study much less is known about the value of their bandgap. McLeod et al ${ }^{14}$ estimated them from soft $\mathrm{X}$-ray emission and X-ray absorption spectroscopy measurements, and obtained values between $0.9 \mathrm{eV}\left(\mathrm{BiMnO}_{3}\right.$ and $\left.\mathrm{BiFeO}_{3}\right)$ and $2.6 \mathrm{eV}\left(\mathrm{BiScO}_{3}\right)$ - the value for $\mathrm{BiFeO}_{3}$ is significantly lower than what others have measured. Computationally, it is well known that standard DFT implementations predict bandgaps that are systematically smaller than experimentally reported ones, and that hybrid implementations such as HSE06 produce much better estimations of the gap $\stackrel{40}{4}$ Because of this, we show in Fig. 8 (top) a comparison between our $\mathrm{DFT}+U$ method-
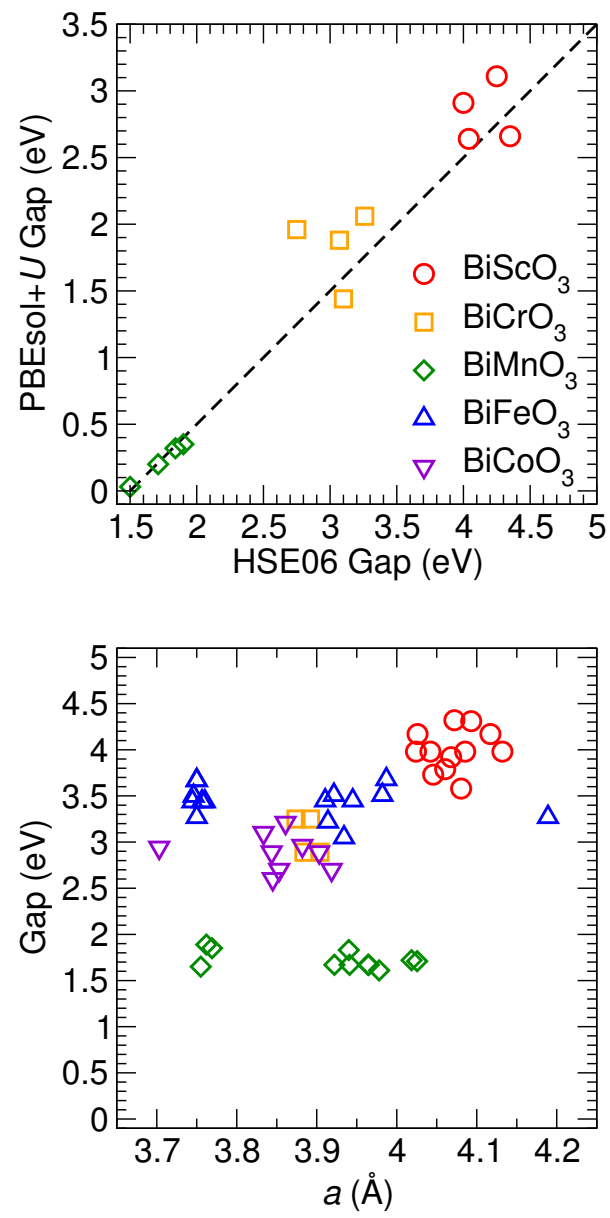

FIG. 8. Top panel: comparison between band gaps computed with DFT $+U$ and with HSE06; the discontinuous line shows that the predictions by the first approach is systematically lower than the one by the second approach, by a shift of around $1.5 \mathrm{eV}$. Bottom panel: value of $\mathrm{DFT}+U$ bandgap for all phases reported in Table III that are energy minima (shifted by $1.5 \mathrm{eV}$ to reflect the most accurate hybrid calculations) as a function of the average between the two closest lattice parameters of their pseudocubic unit cell.

ology and our HSE06 methodology; this shows that the former predicts bandgaps that are around $1.5 \mathrm{eV}$ lower than the latter. With this in mind, Fig. 8 (bottom) shows our DFT $+U$ computations of the band gap of the phases of Table III (shifted by $1.5 \mathrm{eV}$, and done in a reciprocal space grid that is twice more dense in each direction that the one used to optimize the structures). From these results we conclude that, for a given composition, the band gap is not strongly dependent on the particular polymorph. Further, $\mathrm{BiMnO}_{3}$ clearly appears as the most promising material in this family for photovoltaic applications. 


\section{G. Why So Many Polymorphs?}

As mentioned earlier, when similar UPSEX phase searches are applied to prototype ferroelectric perovskites $\mathrm{BaTiO}_{3}$ and $\mathrm{PbTiO}_{3}$ and to Bi-based perovskites $\mathrm{BiXO} \mathrm{O}_{3}$, few minima appear in the former cases and many minima appear in the latter one. How can we understand the difference between these crystals based on the characteristics of the $A$ and $B$ cations?

One particularity of the Bi-based perovskites is the lone pair of $6 s$ electrons of $\mathrm{Bi}^{3+}$ that overlaps with the O orbitals to attain a lobe-shape form, as shown by, e.g., electron-localization function analyses. $\frac{181956}{\text { The }}$ presence of these lobes breaks the spherical symmetry of the $\mathrm{Bi}^{3+}$ ions, and it causes them to move out of highsymmetry positions - this is how ferroelectricity originates in $\mathrm{BiFeO}_{3}$ and related materials. But those lobes can be accommodated in many other ways (paying small energy prices) that give rise to local minima of the energy illustrated in Table II for $\mathrm{BiFeO}_{3}$.

$\mathrm{Ba}$ is an spherical ion, and therefore in $\mathrm{BaTiO}_{3}$ it will not add variety to the structures of this perovskite. However, $\mathrm{Pb}$ does have a $6 s$ electron pair, so the case of $\mathrm{PbTiO}_{3}$ must be analyzed further. There are two main differences between $\mathrm{Bi}$ and $\mathrm{Pb}$ : (i) $\mathrm{Bi}^{3+}$ is smaller (Shannon radius of $1.17 \AA$ at maximum coordination reported ${ }^{\sqrt[57]{7}}$ ) than $\mathrm{Pb}^{2+}$ (Shannon radius of $1.49 \AA$ ); and (ii) $\mathrm{Bi}^{3+}$ is more electronegative than $\mathrm{Pb}^{2+}$ (2.02 versus 1.87 in the Pauling scale $\left.{ }^{61}\right)$. The $s / p$ mixing leading to the active lone pair arises from the interaction with the $p$ orbitals of oxygen; since $\mathrm{Bi}^{3+}$ is more electronegative there should be a better match in this respect, and since $\mathrm{Bi}^{3+}$ is smaller it should adapt better to the electronic requirements of the metal-oxygen lattice to distort. In any case, it is an oversimplification to assume that the energy surface of $\mathrm{PbTiO}_{3}$ has one unique feature (its tetragonal minimum); for example, it is known that its cubic phase has tilt instabilities, which only decay into an untilted phase as strain coupling occurs.6263

The differences between $\mathrm{BaTiO}_{3}, \mathrm{PbTiO}_{3}$, and $\mathrm{Bi} X \mathrm{O}_{3}$ can be therefore attributed mainly to the uniqueness of $\mathrm{Bi}^{+3}$ 's lone pair, but the properties of the transitionmetal cation also play a role. In particular, they must be responsible for the variations in the structures of the $\mathrm{Bi} X \mathrm{O}_{3}$ oxides, where the formal $d$ electron count is the main difference between transition-metal atoms that in some cases are very similar in size (the Shannon radii $\sqrt{57}$ of $\mathrm{Sc}^{3+}, \mathrm{Cr}^{3+}, \mathrm{Mn}^{3+}, \mathrm{Fe}^{3+}$, and $\mathrm{Co}^{3+}$ are $0.745 \AA, 0.615 \AA$, $0.645 \AA$, $0.645 \AA$, and $0.61 \AA$, respectively).

For a $d^{0}$ perovskite $\left(\mathrm{BaTiO}_{3}, \mathrm{PbTiO}_{3}, \mathrm{BiScO}_{3}\right)$ distortions away for the ideal cubic structure can stabilize the system because only the metal-oxygen bonding levels are filled, and none of the metal-based orbitals (which are metal-oxygen antibonding) are occupied. In $\mathrm{BaTiO}_{3}$ atoms move most favorably towards a triangular face of a $\mathrm{O}_{6}$ octahedron, resulting in its $R 3 \mathrm{~m}$ rhombohedral phase. The presence of a lone pair and a large $\mathrm{Pb}^{+2}$ cation changes this balance and makes a tetragonal phase more stable in $\mathrm{PbTiO}_{3}$. A smaller ion as $\mathrm{Bi}^{+3}$ results, as mentioned above, in more freedom for the ion to explore its surroundings, and consequently in more phases.

Once the $d$ electron count increases, more possibilities open. For example, vanadates $\left(d^{1}\right)$ have a tendency to exhibit a square pyramidal coordination, 64 and they do so in a perovskite like $\mathrm{PbVO}_{3}, \frac{65}{,}$ giving rise to a supertetragonal structure. $\mathrm{BiCoO}_{3}$ assumes a related high-spin $\mathrm{d}^{6}$ configuration (a spherically symmetric high-spin $d^{5}$ configuration plus a $d^{1}$ one) and therefore exhibits the same type of $P 4 \mathrm{~mm}$ structure in its ground state. Now of course the other cation is $\mathrm{Bi}^{3+}$, and thus many more possibilities arise.

Systems like $\mathrm{BiScO}_{3}\left(d^{0}\right), \mathrm{BiFeO}_{3}$ (high spin $d^{5}$ ), and even $\mathrm{BiCrO}_{3}$ (high spin $d^{3}$, occupying all $t_{2 g}$ levels with one electron) contain a transition-metal atom with some sort of electronic closed shell, so in principle they should be quite similar; indeed we see some of the structures in Table IIIbeing shared for these materials. However, finer effects are also at play, involving $\mathrm{O}_{6}$ rotations that are mainly combinations of three types: $\left(a^{-} a^{-} a^{0}\right),\left(a^{0} a^{0} c^{+}\right)$, and $\left(a^{0} a^{0} c^{-}\right)$. These add variety to the structures, and result in phases like $C 2 / c$ being also quite low in energy. This is because $C 2 / c$ combines the very energetically favourable $\left(a^{-} a^{-} c^{+}\right)$rotations with parallel and antiparallel $\mathrm{Bi}$ displacements to accommodate the lone pair lobes. In this respect, it represents a compromise between the paraelectric Pnma phase and the strongly polar $R 3 c$ phase.

The Jahn-Teller active ions $\mathrm{Mn}^{+3}\left(d^{4}\right)$ and $\mathrm{Co}^{+3}\left(d^{6}\right)$ add extra possible distortions, reflected for example in the breaking of symmetry that affects the phase that is the ground state of $R 3 c$ and energetically competitive in $\mathrm{BiCoO}_{3}$ and $\mathrm{BiMnO}_{3}$, but with space group $C c$.

\section{CONCLUSIONS}

In this article we report tens of metastable structures of $\mathrm{BiXO}_{3}(X=\mathrm{Sc}, \mathrm{Cr}, \mathrm{Mn}, \mathrm{Fe}, \mathrm{Co})$ compounds. These are minima of the energy within $200 \mathrm{meV} / \mathrm{fu}$ or less of the ground state, as computed using methods based on DFT (more minima exist at higher energy differences). This large degree of polymorphism is related in part to the same mechanism that is responsible for large values of the polarization in many of them: the lone electron pair of the $\mathrm{Bi}^{3+}$ ion. ${ }^{1819 \mid 56}$ These materials can accommodate those pairs in many combinations of polar and antipolar displacements that result in many energy minima. In addition to this, three other factors add variety to these structures: (1) several available $\mathrm{O}_{6}$ rotation patterns, mainly based on combinations of $\left(a^{-} a^{-} c^{0}\right),\left(a^{0} a^{0} c^{+}\right)$, and $\left(a^{0} a^{0} c^{-}\right)$; (2) the possibility of breaking one of the $X-\mathrm{O}$ bonds to create stable supertetragonal phases in $\mathrm{BiMnO}_{3}, \mathrm{BiFeO}_{3}$, and $\mathrm{BiCoO}_{3}$, and inverse supertetragonal phases in $\mathrm{BiFeO}_{3}$; and (3) Jahn-Teller distortions in $\mathrm{BiMnO}_{3}$ and $\mathrm{BiCoO}_{3}$.

In this way, we see that the variety of lowest-energy 
states in this family is just a reflection of the fine balance between the energies involved in the cation displacements and the octahedra rotations. Our calculations show that the reported experimental structures of these materials actually exist in all of them (with the exception of the variations of the supertetragonal structure of $\mathrm{BiCoO}_{3}$, which do not exist in $\mathrm{BiScO}_{3}$ and $\mathrm{BiCrO}_{3}$ as minima). When the materials are grown, the small energy differences involved translate into the dramatic structural differences seen. Epitaxial strain and pressure may be used to shift this balance and favor different polymorphs (including polar ones), as it has been done already in supertetragonal $\mathrm{BiFeO}_{3}{ }^{7 / 66}$

While many calculations based on DFT agree that the ground states of $\mathrm{BiCoO}_{3}$ and $\mathrm{BiFeO}_{3}$ are the ones found experimentally, the situation for $\mathrm{BiScO}_{3}, \mathrm{BiCrO}_{3}$, and $\mathrm{BiMnO}_{3}$ is not so simple. Our calculations using the accurate HSE06 hybrid functional show that the experimentally reported $C 2 / c$ phase is in competition with a similar phase where the primitive unit cell doubles and an extra $\left(a^{0} a^{0} c^{+}\right) \mathrm{O}_{6}$ rotation appears. In the case of $\mathrm{BiCrO}_{3}$, this new phase is slightly lower in energy, and so are the R3c and Pnma phases.

As part of this research, we have also identified local minima of the energy in $\mathrm{BiMnO}_{3}$ where charge ordering is present- $\mathrm{Mn}^{2+}$ and $\mathrm{Mn}^{+4}$ ions alternate in a rocksalt pattern inside the $\mathrm{O}_{6}$ octahedra. These phases are not far in energy from the ground state, and they might be stabilized by growing $\mathrm{BiMnO}_{3}$ on double perovskites that favor this charge ordering.

A detailed study of the $P m c 2_{1}$ inverse supertetragonal phase found here as a bulk minimum adds to the evidence that $\mathrm{BiFeO}_{3}$ might grow into this polymorph on square-symmetry substrates with in-plane lattice parameter around $4.2 \AA$; this was proposed earlier by Yang et $a l ., 50$ who identified this polymorph as a favored configuration in epitaxial films under large tensile strains. In other materials of the family this is also a special point of the energy surface, and therefore amenable to stabilization.

Finally, calculations of band gaps for these structures show that $\mathrm{BiMnO}_{3}$ is the most promising of these materials for optical applications, since it has the lowest band gap of the family - between $1.5 \mathrm{eV}$ and $2.0 \mathrm{eV}$ according to our HSE06 hybrid calculations.

\section{ACKNOWLEDGEMENTS}

O.D. acknowledges funding from the Israel Science Foundation through Grants 1814/14 and 2143/14. Work in Bellaterra was supported by MINECO (Spain) through Grant FIS2015-64886-C5-3-P as well as the Severo Ochoa Centers of Excellence Programs under Grant SEV-2015-0496, and by Generalitat de Catalunya (2017SGR1506). J.I. was supported by the Luxembourg National Research Fund through Grant No. P12/4853155 COFERMAT.
* Corresponding author: dieguez@tau.ac.il

1 J. Wang, J.B. Neaton, H. Zheng, V. Nagarajan, S.B. Ogale, B. Liu, D. Viehland, V. Vaithyanathan, D.G. Schlom, U.V. Waghmare, N.A. Spaldin, K.M. Rabe, M. Wuttig, and R. Ramesh, Science 299, 1719 (2003).

2 G. Catalan and J. F. Scott, Adv. Mater. 21, 2463 (2009).

3 N. Hur, S. Park, P.A. Sharma, J.S. Ahn, S. Guha, and S.-W. Cheong, Nature 429, 392 (2004).

4 Y.-H. Chu, L.W. Martin, M.B. Holcomb, M. Gajek, S.J. Han, Q. He, N. Balke, C.-H. Yang, D. Lee, W. Hu, Q. Zhan, P.-L. Yang, A. Fraile-Rodriguez, A. Scholl, S.X. Wang, and R. Ramesh, Nat. Mater. 7, 478 (2008).

5 J. Seidel, L.W. Martin, Q. He, Q. Zhan, Y.-H. Chu, A. Rother, M.E. Hawkridge, P. Maksymovych, P. Yu, M. Gajek, N. Balke, S.V. Kalinin, S. Gemming, F. Wang, G. Catalan, J.F. Scott, N.A. Spaldin, J. Orenstein, and R. Ramesh, Nat. Mater. 8, 229 (2009).

6 S.Y. Yang, J. Seidel, S.J. Byrnes, P. Shafer, C.-H. Yang, M.D. Rossell, P. Yu, Y.-H. Chu, J.F. Scott, J.W. Ager, L.W. Martin, and R. Ramesh, Nature Nanotechnology 5, 143 (2010).

7 R.J. Zeches, M.D. Rossell, J.X. Zhang, A.J. Hatt, Q. He, C.H. Yang, A. Kumar, C.H. Wang, A. Melville, C. Adamo, G. Sheng, Y.-H. Chu, J.F. Ihlefeld, R. Erni, C. Ederer, V. Gopalan, L.Q. Chen, D.G. Schlom, N.A. Spaldin, L.W. Martin, and R. Ramesh, Science 326, 977 (2009).

8 C. Michel, J.M. Moreau, G.D. Achenbach, R. Gerson, and W.J. James, Solid State Communications 7, 701 (1969).
9 F. Kubel and H. Schmid, Acta Crystallogr. B 46, 698 (1990).

10 A.A. Belik, Journal of Solid State Chemistry 195, 32 (2012).

11 M. Guennou, M. Viret, and J. Kreisel, C. R. Physique 16, 182 (2015).

12 M.W. Lufaso and P.M. Woodward, Acta Crystallogr. Sect. B 57, 725 (2001).

13 P. Chen, M.N. Grisolia, H. J. Zhao, O.E. GonzálezVázquez, L. Bellaiche, M. Bibes, B.-G. Liu, and J. Íñiguez, Phys. Rev. B 97, 024113 (2018).

14 J.A. McLeod, Z.V. Pchelkina, L.D. Finkelstein, E.Z. Kurmaev, R.G. Wilks, A. Moewes, I.V. Solovyev, A.A. Belik, E. Takayama-Muromachi, Phys. Rev. B 81144103 (2010).

15 P. Baettig, R. Seshadri, and N.A. Spaldin, J. Am. Chem. Soc. 1299854 (2007).

16 Y. Uratani, T. Shishidou, F. Ishii, and T. Oguchi, Jpn. J. Appl. Phys. 44, 7130 (2005).

17 P. Baettig, C. Ederer, and N. Spaldin, Phys. Rev. B 72, 214105 (2005).

18 P. Ravindran, R. Vidya, A. Kjekshus, H. Fjellvg, and O. Eriksson, Phys. Rev. B 74, 224412 (2006).

19 O. Diéguez, O. E. González-Vázquez, J. C. Wojdeł, and J. Íñiguez, Phys. Rev. B 83, 094105 (2011).

20 O. Diéguez and J. Íñiguez, Phys. Rev. B 91, 184113 (2015).

21 O. Diéguez and J. Íñiguez, Phys. Rev. Lett. 107, 057601 (2011). 
22 D.C. Arnold, IEEE Trans. Ultrason. Ferroelectr. Freq. Control, 62, 62 (2015).

23 D.V. Karpinsky, I.O. Troyanchuk, M. Tovar, V. Sikolenko, V. Efimov, and A.L. Kholkin, Journal of Alloys and Compounds 555, 101 (2013).

24 X.X. Shi, X.Q. Liu, and X.M. Chen, Journal of Applied Physics 119, 064104 (2016).

25 O.E. González-Vázquez, J.C. Wojdeł, O. Diéguez, and J. Íñiguez, Phys. Rev. B 85, 064119 (2012).

26 S. Prosandeev, W. Dawei, R. Wei, J. Íñiguez, and L. Bellaiche, Adv. Funct. Mater. 23, 234 (2013).

27 A.R. Oganov and C.W. Glass, J. Chem. Phys., 124, 244704 (2006).

28 C.W. Glass, A.R. Oganov and N. Hansen, Comp. Phys. Comm., 175, 713, (2006)

29 A.O. Lyakhov, A.R. Oganov, H.T. Stokes and Q. Zhu, Comp. Phys. Comm., 184, 1172-1182, (2013).

30 G. Kresse and J. Hafner, Phys. Rev. B 47558 (1993); G. Kresse and J. Hafner, Phys. Rev. B 49, 14251 (1994); G. Kresse and J. Furthmüller, J Comput. Mat. Sci. 6, 15 (1996); G. Kresse and J. Furthmüller, Phys. Rev. B 54, 11169 (1996); G. Kresse, Thesis, 1993, Technische Universit at Wien.

31 P. Hohenberg and W. Kohn, Phys. Rev. 136, B864 (1964).

${ }^{32}$ W. Kohn and L. J. Sham, Phys. Rev. 140, A1133 (1965).

33 B. Himmetoglu, A. Floris, S. de Gironcoli, and M. Cococcioni, Int. J. Quantum Chem. 114, 14 (2014).

34 S.L. Dudarev, G.A. Botton, S.Y. Savrasov, C.J. Humphreys, and A.P. Sutton, Phys. Rev. B 57, 1505094105 (1998).

35 A.I. Liechtenstein, V.I. Anisimov and J. Zaane, Phys. Rev. B 52, R5467 (1995).

36 O. Diéguez and J. Íñiguez, Phys. Rev. B 95, 085129 (2017).

37 P. E. Blöchl, Phys. Rev. B. 50, 17953 (1994).

${ }^{38}$ G. Kresse and D. Joubert, Phys. Rev. B. 59, 1758 (1999).

39 P. Pulay, Chem. Phys. Lett. 73, 393 (1980).

${ }^{40}$ A. V. Krakau, O. A. Vydrov, A. F. Izmaylov, and G. E. Scuseria, J. Chem. Phys. 125, 224106 (2006).

41 A. Stroppa and S. Picozzi, Phys. Chem. Chem. Phys. 12, 5405 (2010).

42 A.R. Oganov and M. Valle, J. Chem. Phys 130, 104504 (2009).

43 S. Trolier-McKinstry, M.D. Biegalski, J. Wang, A.A. Belik, E. Takayama-Muromachi, and I. Levin, J. Appl. Phys. 104, 044102 (2008).

44 A.M. Glazer, Acta Cryst. B28, 3384 (1972); A.M. Glazer, Acta Cryst. A31, 756 (1974).

45 K.M. Rabe, in Functional Metal Oxides: New Science and Novel Applications, edited by S. Ogale, V. Venkateshan, and M.G. Blamire (Wiley, Hoboken, NJ, 2013)

46 B. Xu, J. Íñiguez, and L. Bellaiche, Nature Communications 8, 15682 (2017).

47 D. Kan, L. Pálová, V. Anbusathaiah, C.J. Cheng, S. Fujino, N. Valanoor, K.M. Rabe, and I. Takeuchi, Adv. Funct. Mater. 20, 1108 (2010).

48 The Isotropy software suite is available at http://iso.byu.edu

49 C. Escorihuela-Sayalero, O. Diéguez, and J. Íñiguez, Phys. Rev. Lett. 109, 247202 (2012).

50 Y. Yang, W. Ren, M. Stengel, X.H. Yan, and L. Bellaiche, Phys. Rev. Lett. 109, 057602 (2012).

51 O. Diéguez, P. Aguado-Puente, J. Junquera, and J. Íñiguez, Phys. Rev. B 87, 024102 (2013).

52 S. Farokhipoor, C. Magén, S. Venkatesan, J. Íñiguez, C.J.M. Daumont, D. Rubi, E. Snoeck, M. Mostovoy, C. de Graaf, A. Müller, M. Döblinger, C. Scheu, and B. Noheda, Nature 515, 379 (2014).

${ }^{53}$ X. Martí, P. Ferrer, J. Herrero-Albillos, J. Narvaez, V. Holy, N. Barrett, M. Alexe, and G. Catalan, Phys. Rev. Lett. 106, 236101 (2011).

54 C.N.R. Rao, A. Arulraj, P.N. Santosh, and A.K. Cheetham, Chem. Mater. 10, 2714 (1998).

55 S. Ishiwata, M. Azuma, M. Takano, E. Nishibori, M. Takata, M. Sakata, and K. Kato, J. Mater. Chem. 12, 3733 (2002).

56 R. Seshadri and N.A. Hill, Chem. Mater. 13, 2892 (2001).

57 R.D. Shannon, Acta Cryst. A32, 751 (1976).

58 G. King and P.M. Woodward, J. Mater. Chem. 20, 5785 (2010)

59 J. Kreisel, M. Alexe, and P.A. Thomas, Nature Materials 11, 260 (2012).

${ }^{60}$ I. Grinberg, D.V. West, M. Torres, G. Gou, D.M. Stein, L. Wu, G. Chen, E.M. Gallo, A.R. Akbashev, P.K. Davies, J.E. Spanier, and A.M. Rappe, Nature 503, 509 (2013).

61 A.L. Allred, J. Inorg. Nucl. Chem. 17, 215 (1061).

62 Ph. Ghosez, E. Cockayne, U.V. Waghmare, and K.M. Rabe, Phys. Rev. B 60, 836 (1999).

63 J.C. Wojde, P. Hermet, M.P. Ljungberg, Ph. Ghosez, and J. Íñiguez, J. Phys.: Condens. Matter 25, 305401 (2013).

64 P.Y. Zavalij and M.S. Whittingham, Acta Cryst. B 55, 627 (1999).

65 A.A. Belik, M. Azuma, T. Saito, Y. Shimakawa, and M. Takano, Chem. Mater. 17, 269 (2005).

${ }^{66}$ H. Béa, B. Dupé, S. Fusil, R. Mattana, E. Jacquet, B. Warot-Fonrose, F. Wilhelm, A. Rogalev, S. Petit, V. Cros, A. Anane, F. Petroff, K. Bouzehouane, G. Geneste, B. Dkhil, S. Lisenkov, I. Ponomareva, L. Bellaiche, M. Bibes, and A. Barthélmy, Phys. Rev. Lett. 102, 217603 (2009). 